\title{
Multiplicity among chemically peculiar stars
}

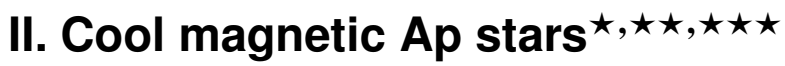

\author{
F. Carrier ${ }^{1}$, P. North ${ }^{2}$, S. Udry ${ }^{1}$, and J. Babel ${ }^{3}$
}

\author{
1 Observatoire de Genève, 1290 Sauverny, Switzerland \\ 2 Institut d'Astronomie de l'Université de Lausanne, 1290 Chavannes-des-bois, Switzerland \\ 3 Office Fédéral de la Statistique, Espace de l'Europe 10, 2010 Neuchâtel, Switzerland
}

Received 16 May 2002 / Accepted 12 July 2002

\begin{abstract}
We present new orbits for sixteen Ap spectroscopic binaries, four of which might in fact be Am stars, and give their orbital elements. Four of them are SB2 systems: HD 5550, HD 22128, HD 56495 and HD 98088. The twelve other stars are: HD 9996, HD 12288, HD 40711, HD 54908, HD 65339, HD 73709, HD 105680, HD 138426, HD 184471, HD 188854, HD 200405 and HD 216533. Rough estimates of the individual masses of the components of HD 65339 (53 Cam) are given, combining our radial velocities with the results of speckle interferometry and with Hipparcos parallaxes. Considering the mass functions of 74 spectroscopic binaries from this work and from the literature, we conclude that the distribution of the mass ratio is the same for cool Ap stars and for normal G dwarfs. Therefore, the only differences between binaries with normal stars and those hosting an Ap star lie in the period distribution: except for the case of HD 200405, all orbital periods are longer than (or equal to) 3 days. A consequence of this peculiar distribution is a deficit of null eccentricities. There is no indication that the secondary has a special nature, like e.g. a white dwarf.
\end{abstract}

Key words. stars: chemically peculiar - stars: spectroscopic binaries - stars: fundamental parameters

\section{Introduction}

Ap stars are conspicuous not only because of their strong chemical anomalies, but also because of their strong, large-scale magnetic field (at least in the $\mathrm{Si}$ and $\mathrm{SrCrEu}$ subtypes) and slow rotation. The latter characteristic is associated with a complete lack of Ap stars in binaries with very short orbital periods (i.e. 1.5 days or less), contrarily to normal stars, probably because such systems are synchronized and their components have to rotate fast, which does not seem compatible with the development of chemical peculiarities. One could also think of an observational bias as another possible cause, the line widening erasing mild peculiarities; the fact that some Bp or Ap stars do rotate fast $\left(200 \mathrm{~km} \mathrm{~s}^{-1}\right)$ does not support this explanation, however. But, in addition to the fact that tidal synchronisation will preclude the existence of Ap stars in short period systems, one may reasonably expect that some special conditions are needed to form an Ap star, and that these conditions might

Send offprint requests to: $\mathrm{P}$. North, e-mail: fabien.carrier@obs.unige.ch

* Based on observations collected at the Observatoire de HauteProvence (CNRS), France.

$\star \star$ Tables 1 to 3 are only available in electronic form at the CDS via anonymous ftp to cdsarc.u-strasbg.fr $(130.79 .128 .5)$ or via http://cdsweb.u-strasbg.fr/cgi-bin/qcat?J/A+A/394/151 $\star \star \star$ Appendix $B$ is only available in electronic form at http://www. edpsciences.org leave their blueprint not only in the magnetic field and slow rotation, but also in the frequency and orbital elements of binaries. One important purpose of this paper is precisely to explore this possibility.

The first, systematic search for binaries among Ap stars has been done by Abt \& Snowden (1973), who examined 62 bright northern stars and concluded to a low rate of binaries (20 percent), except for $\mathrm{HgMn}$ stars (43 percent). Aikman (1976) increased the sample of $\mathrm{HgMn}$ stars from 15 to 80 and confirmed the rate found by Abt $\&$ Snowden, since he found 49 percent, which is very close to the result of Jaschek \& Gomez

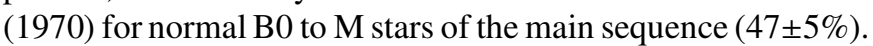

There has been no systematic review of multiplicity among Ap stars since the work of Gerbaldi et al. (1985, hereafter GFH85), apart from some attempts by Budaj (1995, 1996, 1997) to interpret the role that a binary companion might have in the appearance of chemical peculiarities of both Am and Ap stars. According to GFH85, the rate of binaries tends to be rather small among the He-weak and Si stars. For the coolest Ap stars, as well as for the HgMn stars, this rate behaves in the same way as for normal stars. Moreover, the magnetic Ap stars show a strong deficit of SB2 binaries: only two SB2's containing a magnetic Ap star had been well studied before the CORAVEL observations: HD 55719 (Bonsack 1976) and HD 98088 (Abt et al. 1968; Wolff 1974). On the other hand, $\mathrm{HgMn}$ stars, which generally have no significant magnetic field (see, however, Mathys \& Hubrig 1995), are often found in SB2 
systems, and their companion seems to be always an Am star when its effective temperature is below $10000 \mathrm{~K}$ (Ryabchikova 1998). Am stars are also known to be frequently associated with SB2 systems (Abt \& Levy 1985).

A radial-velocity survey of a small number of cool, wellknown magnetic Ap stars has been initiated in 1980 using the CORAVEL scanner (Baranne et al. 1979), and the sample has been extended in 1985 to all stars brighter than $V=8.6$, visible from the northern hemisphere and having Geneva photometry. The purpose was to increase the relatively poor statistics and obtain a better understanding of the role multiplicity might play in the context of chemically peculiar stars. Preliminary results have been published by North (1994), especially the discovery of a long-period SB2 system which has been studied in more details later (HD 59435, Wade et al. 1996, 1999) and the discovery of an SB1 system with a period as short as 1.6 days (HD 200405).

This paper is the second one in a series dedicated to multiplicity among Ap and Am stars. Since Drs. Nicole Ginestet and Jean-Marie Carquillat in Toulouse had independantly measured a few of our programme stars with the same instrument, we had dedicated the first paper of this series to four common stars (North et al. 1998, Paper I), especially the Ap stars HD 8441 and $\beta \mathrm{CrB}$. Here we present the results for all Ap stars measured to date with CORAVEL, with a few additional data from the ELODIE spectrograph (Baranne et al. 1996) which are by-products of a survey of magnetic fields (Babel \& North, in preparation).

\section{Observations and sample}

All radial-velocity observations have been obtained at the Observatoire de Haute-Provence. Most of the data were measured with the CORAVEL scanner attached to the 1-meter Swiss telescope. Although this instrument is optimized for latetype stars, it can still yield very good results on slowly rotating $\mathrm{F}$ and even A stars, especially if their metallic lines are enhanced, as is the case of Ap stars. Originally, the sample was selected according to the following criteria: $T_{\text {eff }} \leqslant 10000 \mathrm{~K} \mathrm{ac}-$ cording to Geneva photometry (stars for which no Geneva photometry existed in 1985 were not retained), $\delta \geqslant-25^{\circ}, m_{V} \leqslant$ 8.6 (except for a few stars lying in the northern galactic polar cap and for candidate high-velocity stars proposed by Jaschek et al. 1983). A total of 1913 radial-velocity observations were made of the 119 programme stars during the period March 1980 through April 1998. Errors for the individual observations are derived following the precepts of Baranne et al. (1979) and are generally below $2.0 \mathrm{~km} \mathrm{~s}^{-1}$ for all stars. The radial-velocity variations are large enough $\left(10 \mathrm{~km} \mathrm{~s}^{-1}<2 \times K<173 \mathrm{~km} \mathrm{~s}^{-1}\right)$ to be clearly significant. In addition to the CORAVEL data, we use here 75 measurements for 72 stars already observed with CORAVEL, obtained with the 1.93-meter telescope at OHP, equipped with the ELODIE spectrograph. This fibre-fed echelle spectrograph has been in operation only since the end of 1993 and has a better precision than the CORAVEL scanner. All but one ELODIE data were gathered during four observing runs from October 1994 to September 1996, which were primarily aimed at determining magnetic fields (Babel et al. 1995;
Babel \& North 1997); the radial velocities are just a by-product of the programme. One additional data was obtained in April 2002 for HD 116114 with ELODIE.

Among all the programme stars, only 20 stars have as yet a precise orbit. $\beta \mathrm{CrB}$ and HD 8441 (as well as the Am star HD 43478) have been published by North et al. (1998), while HD 59435 and HD 81009 have been published by Wade et al. (1996, 1999) and by Wade et al. (2000) respectively; the orbit of HD 73709 was published by Debernardi et al. (2000). The others are presented in the appendix A, as well as an improved orbit of HD 73709. The orbital elements of the 16 stars studied in this article are listed in Table A.1. The individual $R V$ measurements of all stars of the sample ${ }^{1}$, as well as the depths and widths of the correlation dips, are listed in Table 1, which is only available in electronic form at the CDS. The average radial velocities, upper limits to projected rotational velocities and some statistical quantities like $P\left(\chi^{2}\right)$ (see Duquennoy \& Mayor 1991 for a definition of $P\left(\chi^{2}\right)$ ), are given in Table 2 (only available in electronic form at the CDS). The list of the 48 stars observed with CORAVEL (in addition to the 119 stars mentioned above), but showing no measurable correlation dip is given in Table 3 (only available in electronic form at the CDS).

\section{The correlation dip of magnetic Ap stars}

It is interesting to discuss the properties of the correlation dip in the case of magnetic Ap stars. For normal stars, this dip yields essentially three independant informations, corresponding to the three parameters of the fitted Gaussian: the radial velocity, the width $(\sigma)$ of the dip which is linked with the projected rotational velocity $v \sin i$, and "surface" or equivalent width $W$ of the dip, which depends on the effective temperature and metallicity of the star (at least on the main sequence). A calibration of the width $\sigma$ in terms of $v \sin i$ was proposed by Benz \& Mayor $(1981,1984)$ who showed that a temperature (or colour index $B-V$ ) term has to be introduced. This calibration has been verified in the range of spectral types F6 to M0 (for luminosity class V), but has been also applied to our hotter stars, implying a slight extrapolation. Mayor (1980) first showed that the dip's equivalent width $W$ could be efficiently calibrated in terms of metallicity $[\mathrm{Fe} / \mathrm{H}]$, provided a good indicator of $T_{\text {eff }}$ be known, e.g. a colour index like Johnson's $B-V$ or Geneva $B 2-V 1$. An explicit calibration of $W$ for Am to $G$ stars in terms of $[\mathrm{Fe} / \mathrm{H}]$ was first proposed by North \& Duquennoy (1991) and yielded good results (the rms scatter of the difference between the spectroscopic and CORAVEL $[\mathrm{Fe} / \mathrm{H}]$ amounted to no more than $0.12 \mathrm{dex}$ ), although the sources of spectroscopic $[\mathrm{Fe} / \mathrm{H}]$ values were heterogeneous. Later, Pont (1997) based a similar calibration on the very large and homogeneous sample of spectroscopic metallicities of Edvardsson et al. (1993) and obtained

\footnotetext{
Except for HD 43478, HD 59435, HD 81009 and $\beta \mathrm{CrB}$ which were already published. The data for HD 8441 are given anew in Tables 1 and 2, because a few additional measurements have been made and all measurements are now in the ELODIE $R V$ system; these data may be useful since HD 8441 is a triple system whose longer period is not yet known.
} 
an excellent result, the rms scatter of the differences between spectroscopic and CORAVEL [Fe/H] values being only 0.074 .

In the case of magnetic Ap stars, the situation is complicated in three ways:

- The abundances are not standard (i.e. solar scaled), but some elements are considerably enhanced - especially the rare earths in cool Ap stars considered here - while others are underabundant (e.g. He, C, N, O) in the atmosphere. Therefore, one can no more, in principle, consider $[\mathrm{Fe} / \mathrm{H}]$ as a meaningful metallicity indicator. Nevertheless, in practice $W$ should remain strongly correlated with $[\mathrm{Fe} / \mathrm{H}]$, since most lines of CORAVEL's mask are neutral iron lines, or at least lines of iron-peak elements;

- The abundances are not distributed uniformly over the stellar surface, but are often concentrated in patches whose positions depend on the geometry of the magnetic field. This implies that potentially, all three parameters of the correlation dip may vary as a function of time, according to the rotation of the star. Significant changes of the width and depth of the dip are indeed observed in some cases, but remain often negligible. Radial velocity variations due to abundance patches or spots sometimes occur as well, when the star has a significant $v \sin i$, since spots with enhanced lines of $\mathrm{Fe}$ peak elements will contribute most to the correlation dip. Thus, in some favourable cases, one can determine the rotational periods;

- The magnetic field may widen the dip through the Zeeman effect and also enhance its equivalent width through Zeeman intensification of the lines. As a result, those stars which have a strong surface field $H_{\mathrm{s}}$ (the disk-averaged modulus of the field, see e.g. Preston 1971) yield so large a dip that they mimick normal stars with a much larger $v \sin i$. Therefore, the $v \sin i$ estimates based on the calibration of Benz \& Mayor $(1981,1984)$ can only represent, in general for the magnetic Ap stars, an upper limit to their true $v \sin i$. There is no mean of disentangling $v \sin i$ and $H_{\mathrm{s}}$ in such cases, unless one of these quantities is determined independantly. Though this is true in the case of CORAVEL, such disentangling is possible with the more efficient ELODIE spectrograph when two different masks are used (one selecting lines sensitive to Zeeman effect, the other selecting lines less sensitive to it), as shown by Babel et al. (1995) and by Babel \& North (1997).

\subsection{Zeeman effect}

The latter complication is illustrated in Fig. 1, where the dip width $\sigma$ is plotted as a function of $v \sin i$, which was taken from the literature, first from Preston (1971), then from Abt \& Morrell (1995) and from Renson (1991). Since the interesting range of $v \sin i$ values is between 0 and $30 \mathrm{~km} \mathrm{~s}^{-1}$ and the relative errors are often very large (depending on the spectral resolution, the values given in the literature are sometimes only upper limits), we have taken the minimum value among these three sources, when the star was mentioned in more than one of them. Furthermore, we have estimated the equatorial velocity from the rotational period (given by Renson 1991 or

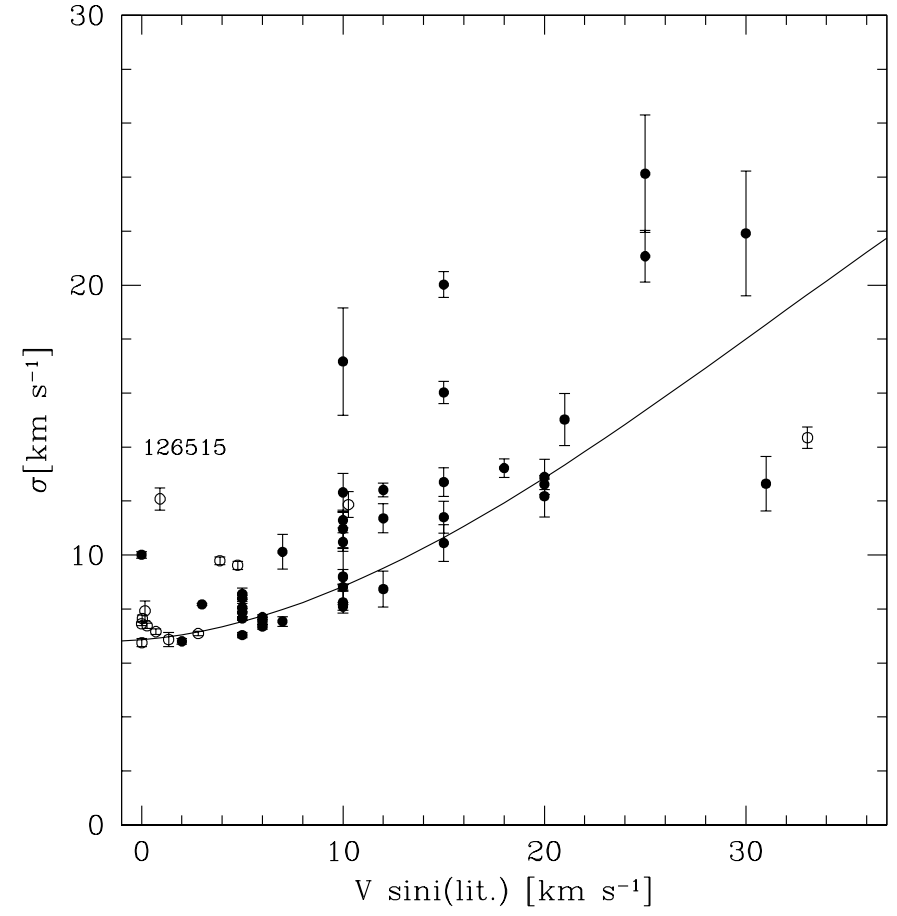

Fig. 1. Width of the Gaussian fitted to the CORAVEL correlation dip as a function of $v \sin i$ of some well studied Ap stars. Full dots: observed $v \sin i$ from the literature. Open dots: $v_{\text {eq }}$ computed from the rotational period and estimated radius through the oblique rotator formula, when it was smaller than the published $v \sin i$ (see text). The full line is the calibration of Benz \& Mayor (1984) for normal F$\mathrm{G}$ stars, extrapolated for effective temperatures relevant to $\mathrm{Ap} \mathrm{SrCrEu}$ stars (around $8000 \mathrm{~K}$ ). Notice the discrepant position of some strongly magnetic stars like HD 126515.

updated by Catalano et al. 1993 and by Catalano \& Renson 1997) and from the radius through the oblique rotator formula $v_{\text {eq }}=50.6 \times R / P_{\text {rot }}$ (with $v_{\text {eq }}$ expressed in $\mathrm{km} \mathrm{s}^{-1}, R$ in solar radii and $P_{\text {rot }}$ in days, see Stibbs 1950), and substituted the measured $v \sin i$ value by $v_{\text {eq }}$ whenever the latter was smaller than the former. The radius was obtained from the Hipparcos parallax and a photometric estimate of the effective temperature, in the way described by North (1998). The quantity $v_{\text {eq }}$ remains an upper limit to the true $v \sin i$ because of the projection effect, but in some cases it is tighter than the observational upper limit. In Fig. 1, the continuous line is the calibration of Benz \& Mayor $(1981,1984)$ for $T_{\text {eff }}=8000 \mathrm{~K}$, i.e. for $B-V=0.183$ according to Eq. (4) of Hauck (1985); it is almost identical to the curve shown in Fig. 3 of Benz \& Mayor (1981). Although many Ap stars fit the normal relation fairly well, some lie significantly above it. Especially conspicuous is the case of HD 126515, which has a well-known rotational period of 130 days (e.g. Mathys et al. 1997) and a strong surface magnetic field which varies between 10 and $17 \mathrm{kG}$ (Preston 1971; Mathys et al. 1997): the large $\sigma$ can evidently not be attributed to $v \sin i$ in this case!

Figure $2 \mathrm{a}$ is the same as Fig. 1, but restricted to stars with a known surface magnetic field, which naturally restricts the sample to slow rotators. The surface field has been taken from Mathys et al. (1997) and from Preston (1971). Figure 2b shows 

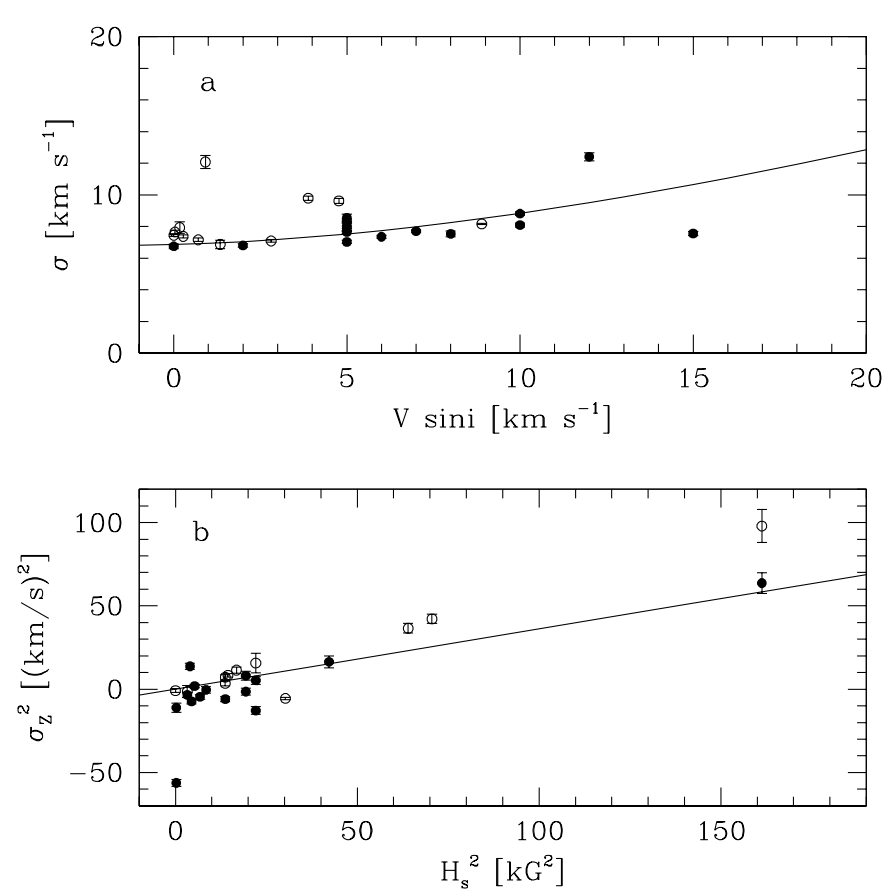

Fig. 2. a) Same as Fig. 1, but only for stars with a known surface magnetic field. b) Quadratic difference between the observed dip width and the value expected from the observed $v \sin i$, using the calibration of Benz \& Mayor $(1981,1984)$, versus $\left\langle H_{\mathrm{s}}\right\rangle^{2}$. The line is the theoretical relation between $\sigma_{Z}^{2}$ and $\left\langle H^{2}\right\rangle$, assuming $\left\langle g_{\text {eff }}\right\rangle=1.0$ (Eq. (5)).

the relation between $\sigma_{Z}^{2}=\sigma^{2}-\sigma_{V}^{2}(v \sin i)$ and the square of the surface field, where $\sigma_{V}^{2}(v \sin i)$ is the $\sigma^{2}$ of the dip expected from the $v \sin i$ through the calibration of Benz \& Mayor and $\sigma$ is the measured, total width. Clearly, a significant correlation emerges, confirming the sensitivity of the correlation dip to Zeeman effect. The points follow roughly the theoretical relation shown by the line, which is determined in the following way.

The wavelength shift $\Delta \lambda$ between the centre of gravity of the $\sigma_{ \pm}$component of a normal Zeeman triplet, and the central wavelength $\lambda_{0}$ of the line without magnetic field is given by

$$
\Delta \lambda=4.67 \times 10^{-13} \lambda_{0}^{2} g_{\text {eff }}\langle H\rangle
$$

where $\lambda_{0}$ is in $\AA, g_{\text {eff }}$ is the effective Landé factor and $\langle H\rangle$, in Gauss, is the modulus of the magnetic field averaged over the visible stellar disk. Dividing this relation by $\lambda_{0}$ and multiplying it with the speed of light $c$, one gets the following expression for the Zeeman shift (expressed in terms of an equivalent Doppler velocity), assuming, furthermore, that we take a mean over a large number of lines:

$\langle\Delta v\rangle=1.40 \times 10^{-7} \lambda_{\text {norm }}\left\langle g_{\text {eff }}\right\rangle\langle H\rangle$

where $\Delta v$ is expressed in $\mathrm{km} \mathrm{s}^{-1}, \lambda_{\text {norm }}$ is a normalisation wavelength (chosen e.g. in the middle of the CORAVEL wavelength range) expressed in $\AA$, and $\left\langle g_{\text {eff }}\right\rangle$ is the effective Landé factor averaged over all spectral lines considered (see Babel et al. 1995).
Assuming now that all sources of broadening of the spectral lines are small enough that the resulting profile remains Gaussian, one can cumulate them by a simple quadratic addition:

$\sigma^{2}=\sigma_{0}^{2}+\sigma_{\text {rot }}^{2}+\left(1.40 \times 10^{-7}\right)^{2} \lambda_{\text {norm }}^{2}\left\langle g_{\text {eff }}^{2}\right\rangle\left\langle H^{2}\right\rangle$

where $\sigma_{0}$ is the sum of the intrinsic and instrumental widths of the lines of a non-rotating and non-magnetic star (it includes the thermal width and depends, therefore, on the effective temperature) and $\sigma_{\text {rot }}$ is only due to projected rotational velocity; both $\sigma_{0}$ and $\sigma_{\text {rot }}$ are in $\mathrm{km} \mathrm{s}^{-1}$. Here, because the Zeeman components remain unresolved, the quantities $g_{\mathrm{eff}}^{2}$ and $H^{2}$ are averaged, instead of $g_{\text {eff }}$ and $H$ as in Eq. (2). The quantity $\left\langle g_{\text {eff }}^{2}\right\rangle$ may be defined as

$\left\langle g_{\mathrm{eff}}^{2}\right\rangle=\frac{\sum_{i=1}^{N}\left(d_{i} \frac{\lambda_{0 i}}{\lambda_{\mathrm{norm}}} g_{\mathrm{eff} i}\right)^{2}}{\sum_{i=1}^{N} d_{i}^{2}}$

where $d_{i}$ is the residual depth of the $i_{\text {th }}$ spectral line.

In Eq. (3), the quantity $\sigma_{0}^{2}+\sigma_{\text {rot }}^{2}$ represents the width of the correlation dip expected for a normal, non-magnetic rotating star, from the calibration of Benz \& Mayor; the third term, which we call $\sigma_{Z}^{2}$, represents the additional contribution of the Zeeman effect. $\sigma$ is the observed total width.

In practice, we chose $\lambda_{\text {norm }}=4300 \AA$, roughly corresponding to the centre of the CORAVEL passband, and assumed $\left\langle g_{\text {eff }}^{2}\right\rangle=1.0 ; \sigma_{Z}$ then becomes:

$\sigma_{Z}^{2}=\sigma^{2}-\left(\sigma_{\text {int }}^{2}+\sigma_{\text {rot }}^{2}\right)=0.362\left\langle H^{2}\right\rangle$

where $H$ is expressed in $\mathrm{kG}$. This result is in rough agreement with the empirical data shown in Fig. 2

\section{Intrinsic variations of the correlation dip}

By "intrinsic", we mean here those variations of the correlation dip which are due to the presence of abundance patches on the surface of the star and to the axial rotation. On the one hand, these variations represent a "noise" regarding the precise determination of the radial velocity of the star as a whole; on the other hand, they represent an additional information which, in some cases, allows an estimate of the rotational period independantly from e.g. photometry. In any case, such variations draw the attention towards those stars which are spectrum variables and may serve as targets for detailed Doppler imaging.

In Fig. 3 are shown the $R V$, dip width and depth variations of the well-known spectrum variable HD 126515. The ephemeris is

$\operatorname{HJD}\left(H_{\mathrm{s}} \max \right)=2437015.000+129.95 E$,

the zero point being defined by Preston (1970) and the period being refined by Mathys et al. (1997). For this particular star, the shape of the dip is clearly changing according to the rotational phase, but the radial velocity remains roughly constant. The rotational velocity is so low that even contrasted abundance patches will not affect $R V$ much. The relation between the surface field and the dip variations are not straightforward: the dip width seems to vary as a double wave, while $H_{\mathrm{s}}$ varies 


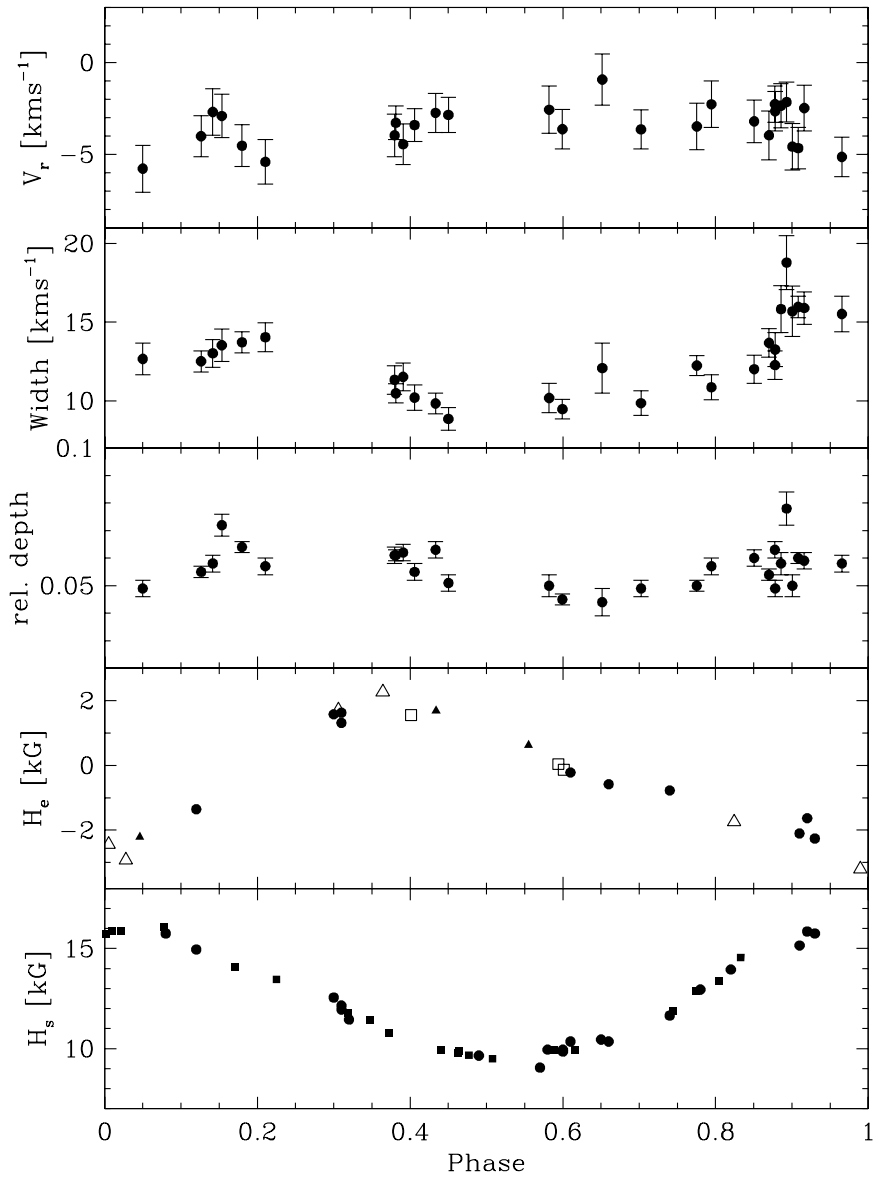

Fig. 3. Variations of the CORAVEL correlation dip as a function of rotational phase for the slowly rotating Ap star HD 126515. These variations are due to abundance patches. The three upper panels show the moment of first order $(R V)$ of the dip, its width and its depth. The 4th panel shows the longitudinal magnetic field (key to symbols: black dots, Preston 1970; open squares, van den Heuvel 1971; open trianges, Mathys 1994; full triangles, Mathys \& Hubrig 1997). The lower panel shows the surface magnetic field (key to symbols: black dots, Preston 1970; full squares, Mathys et al. 1997).

as a single wave. There is a trend for the dip to become wider as $H_{\mathrm{s}}$ increases.

In Fig. 4 are shown the same quantities for the well known Ap star HD 65339 (or 53 Cam), though the fitted orbital radial velocities (see next section) have been subtracted to the observed ones to obtain the variations $\Delta V_{\mathrm{r}}$ due to rotation and abundance patches only. The ephemeris used is from Borra \& Landstreet (1977):

$\operatorname{HJD}($ pos.cross. $)=2435855.652+8.0267 E$.

Here again, the correlation dip appears wider at phases of larger surface magnetic field, as expected from Eq. (6). There are also clear "intrinsic" $R V$ variations in this star, which raises the question of how to discriminate between intrinsic and orbital variations. Such a question is especially acute when trying to estimate the rate of binaries, and was already tackled by Abt \& Snowden (1973). The solution these authors proposed was to use the hydrogen lines instead of the metallic ones: hydrogen being largely predominent, its abundance may be considered

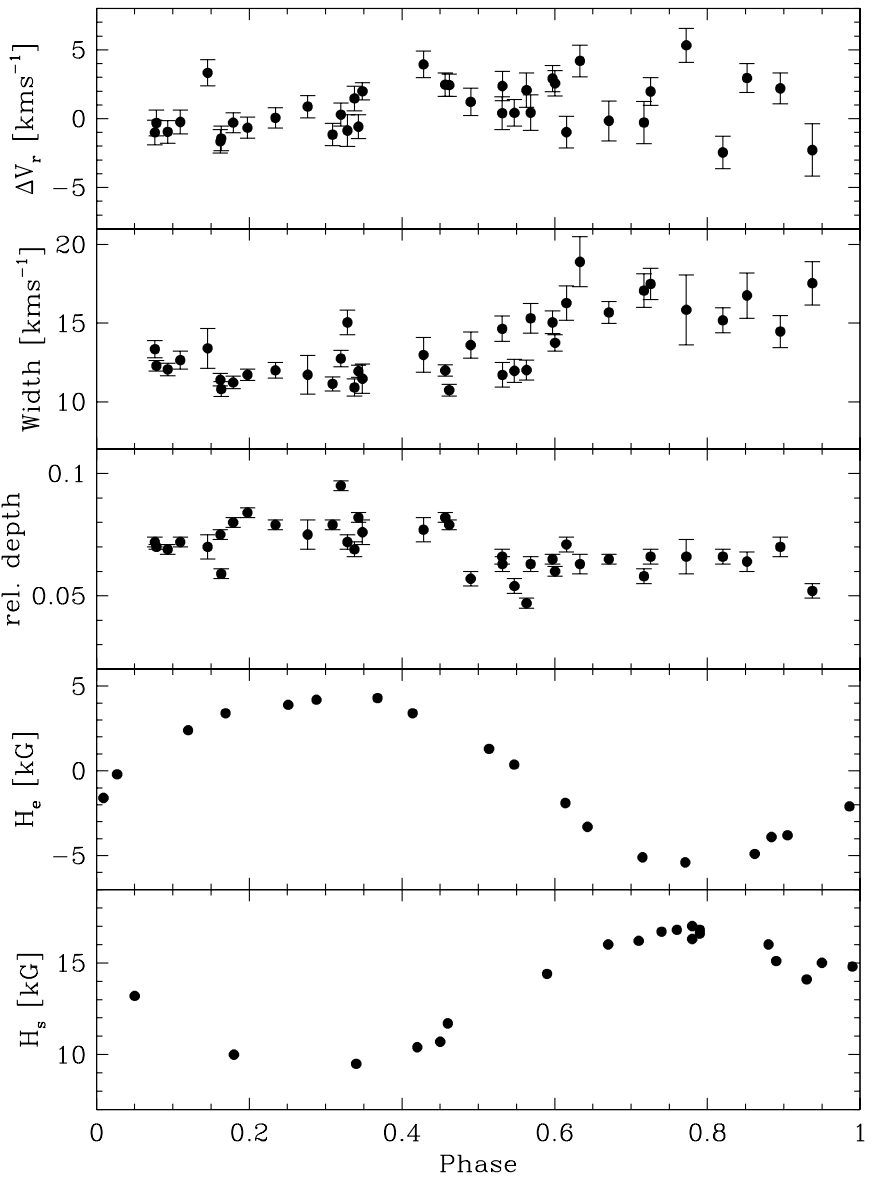

Fig. 4. Same as Fig. 3, but for the Ap star HD 65339. The longitudinal magnetic field is from Borra \& Landstreet (1977). The surface magnetic field is from Huchra (1972).

homogeneous over the whole surface of the star. However, the CORAVEL mask precisely excludes all hydrogen lines, so this solution cannot hold for us. Nevertheless, one observes that those stars presenting an intrinsic $R V$ variation also have broad and shallow dips betraying a relatively fast axial rotation (typically $20 \mathrm{~km} \mathrm{~s}^{-1}$ ). These variations occur on a relatively short timescale, while their amplitude is rather small: this leads to an extremely small mass function, if they are interpreted as due to orbital motion. They might present a problem, in principle, only in the ideal case of an extremely small and strongly contrasted abundance spot, which would yield a sharp, narrow dip with a significant $R V$ variation.

An interesting case which illustrates well the above considerations, is that of the Ap Sr star HD 49976 (HR 2534). It is a conspicuous spectroscopic variable which was studied by Pilachowski et al. (1974). These authors found a rotational period $P=2.976$ days, which was later refined to $P=2.97666 \pm 0.00008$ days by Catalano \& Leone (1994) by means of $u v b y$ photometry. Figure 5, based on the ephemeris

$\mathrm{HJD}($ pos.cross. $)=2441298.76+2.97666 E$

shows a rather well defined, double wave $R V$ variation whose peak-to-peak amplitude is as large as $15 \mathrm{~km} \mathrm{~s}^{-1}$. This is consistent with two spots. There is one maximum around phase 0.3 and another one around phase 0.8 . This is qualitatively 


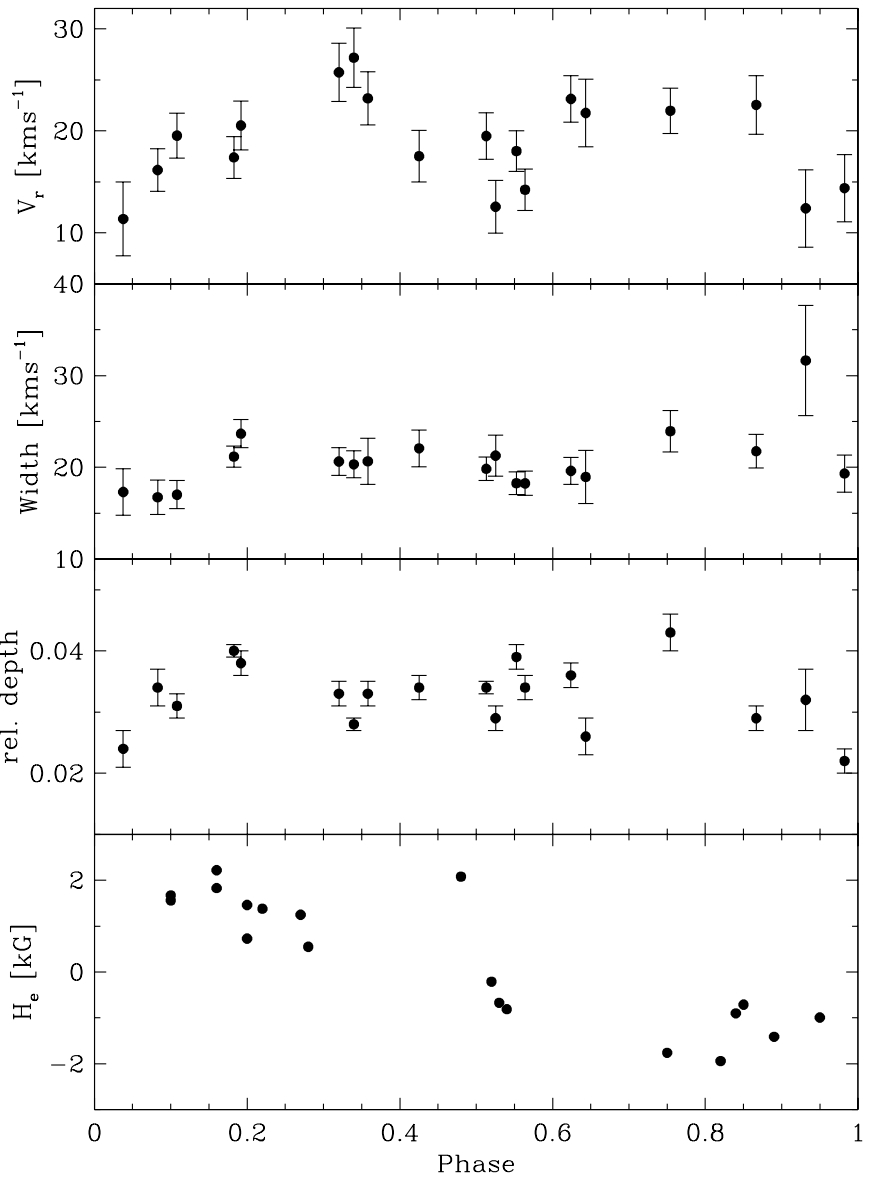

Fig. 5. Same as Fig. 3, but for the Ap star HD 49976. The longitudinal magnetic field is from Pilachowski et al. (1974).

consistent with the $R V$ variation of the $\mathrm{Fe}$ and $\mathrm{Cr}$ lines obtained by Pilachowski et al. (1974, their Fig. 1), though the maxima fall rather near phases 0.4 and 0.9 in their case. The width and depth of the dip are ill-defined because of the large rotational velocity ( $31 \pm 3 \mathrm{~km} \mathrm{~s}^{-1}$ according to these authors).

Finally, we give in Fig. 6 the periodogram of the variation of the dip depth of HD 5797 (V551 Cas), to illustrate how such variations can help to determine rotational periods. We obtain $P_{\text {rot }}=68.02 \pm 0.10$ days, compatible with one of the possible periods proposed by Wolff (1975).

The periodogram is based on the method of Renson (1978), but the reciprocal of his $\theta_{1}$ test has been plotted. There are only twelve observations spanning 5873 days, but it is interesting to see that the other periods suggested by Wolff (1975) as acceptable from her photometric data, 45.5 and 57 days, are clearly rejected in view of our data, even though the latter are scattered on a so long time range. Interestingly, Barzova \& Iliev (1988) and Iliev et al. (1992) have also rejected the two shorter periods on the basis of high resolution spectra, but admit both 67.5 and 69 days as equally possible.

We have verified that our period agrees with the photometric data of Wolff (1975): Renson's method applied to the differential $b$ magnitudes, which seem to have the best $S / N$ ratio, gives $P_{\text {rot }}=68.2 \pm 1.0$ days. Likewise, the $v$ magnitudes, which also have a good $S / N$, give $P_{\text {rot }}=67.6 \pm 0.5$ days. The $y$

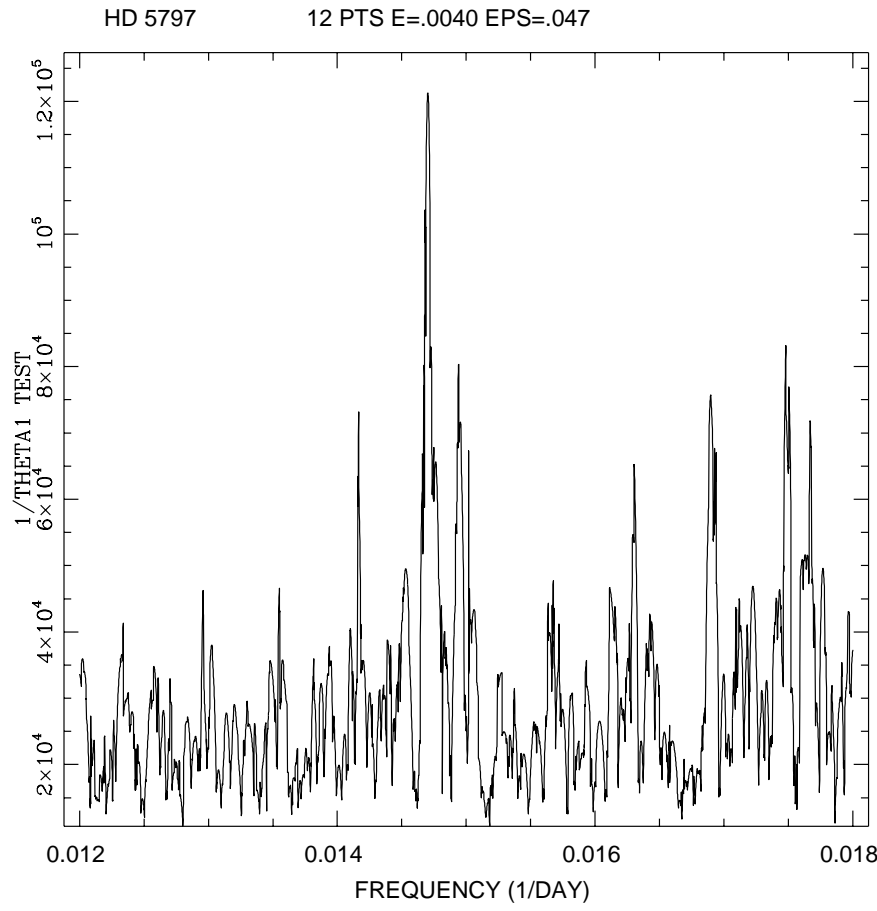

Fig. 6. Periodogram of the variation of the depth of the correlation dip for the Ap CrEuSr star HD 5797. Although it is based on only 12 observations, the peak is well defined and unique in the whole range of frequencies from 0 to 0.03 days $^{-1}$.

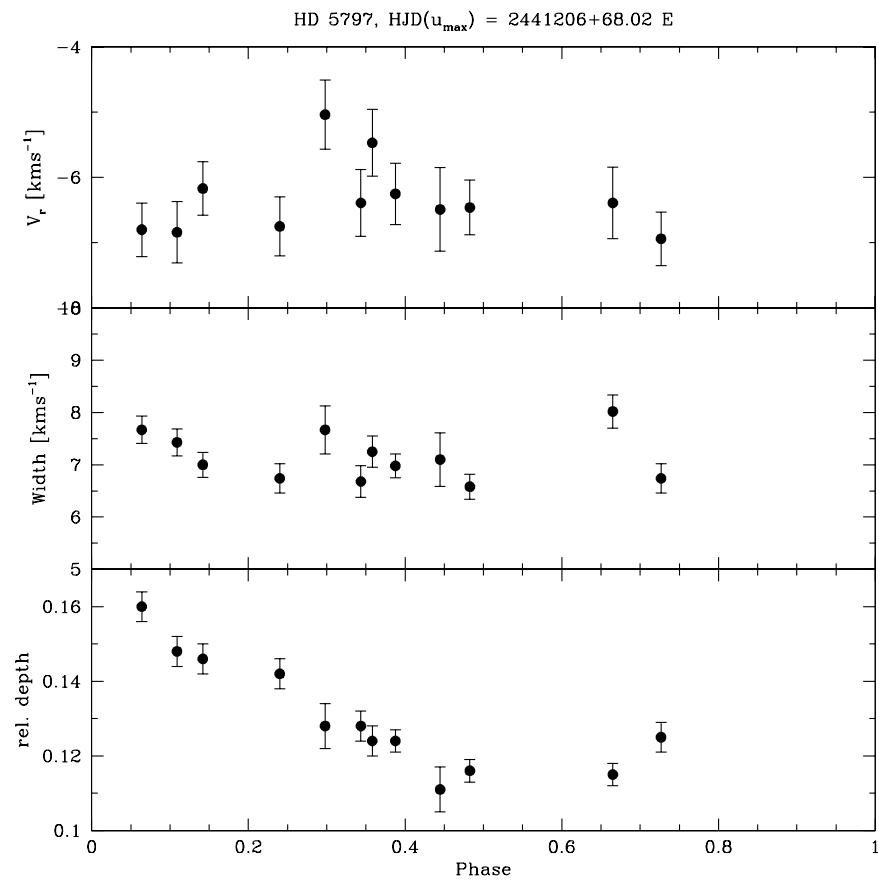

Fig. 7. Same as Fig. 5, but for HD 5797. No magnetic field curve exists for this star.

and $u$ magnitudes, which are more noisy (at least compared to the amplitude of variation) give a best period around 57 days, but are also compatible with the 68 days period. Therefore, our 68.02 days period is quite compatible with existing data and is probably the best estimate available to date. The variation of the three parameters of the correlation dip of HD 5797 is shown 
in Fig. 7. Unfortunately, the phase coverage is not good, but the depth of the dip varies in a very significant way, contrary to the radial velocity and dip width. No longitudinal field curve exist for this star, although Preston (1971) estimated a surface field of $1.8 \mathrm{kG}$.

\section{Statistics of Ap binaries}

\subsection{The sample of binaries}

Our sample is composed of all Ap stars known as spectroscopic binaries. The catalogue of Renson (1991) gives us the Ap stars. However, some stars considered as Am by Renson, have been considered in this work as Ap (HD 56495, HD 73709 and HD 188854). Among these stars, the spectroscopic binaries have been selected from the following sources. Fourteen orbits were determined thanks to the CORAVEL scanner (this paper and North et al. 1998). Lloyd et al. (1995) determined a new orbit for $\theta$ Carinae (HD 93030) and Stickland et al. (1994) for HD 49798. Recently, Leone \& Catanzaro (1999) have published the orbital elements of 7 additional CP stars, two of which are He-strong, two are He-weak, one is Ap Si, one Ap $\mathrm{HgPt}$ and one Ap SrCrEu. Wade et al. (2000) provided the orbital elements of HD 81009. Other data come from the Batten et al. (1989) and Renson (1991) catalogues. Among all Ap, we kept 78 stars with known period and eccentricity, 74 of them having a published mass function.

\subsection{Eccentricities and periods}

The statistical test of Lucy \& Sweeney (1971) has been applied to all binaries with moderate eccentricity, in order to see how far the latter is significant. The eccentricity was put to zero whenever it was found insignificant. We can notice the effect of tidal interactions (Zahn 1977, 1989; Zahn \& Bouchet 1989) on the orbits of Ap stars (see Fig. 8a). Indeed, all orbits with $P$ less than a given value $\left(=P_{\text {circ }}\right)$ are circularized. According to the third Kepler law, a short period implies a small orbit where tidal forces are strong. The period-eccentricity diagram for the Ap stars does not show a well marked transition from circular to eccentric orbits, in the sense that circular as well as eccentric systems exist in the whole range of orbital periods between $P_{\text {circ }}=5$ days to a maximum of about 160 days. The wider circular orbits probably result from systems where the more massive companion once went through the red giant phase; the radius of the former primary was then large enough to circularize the orbit in a very short time. This is quite consistent with the synchronization limit for giant stars of 3 to $4 M_{\odot}(P \sim 150$ days, Mermilliod \& Mayor 1996).

An upper envelope seems well-defined in the $e$ vs. $\log P$ diagram, especially for $\log P \lessgtr 2$, although four points lie above it. The leftmost of these, with $(\log P, e)=(0.69,0.52)$ has a rather ill-defined orbit ${ }^{2}$. Whether this envelope has any significance remains to be confirmed with a larger sample than presently available.

221 Her (HD 147869) has been measured by Harper (1931) and has a relatively small amplitude.

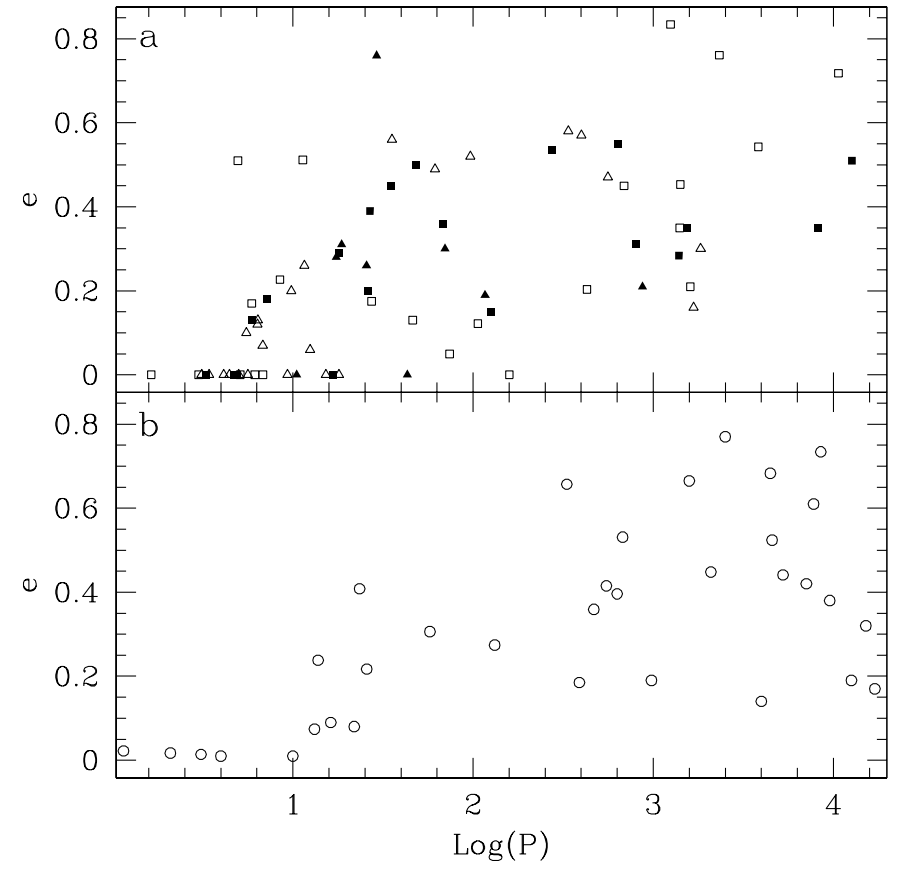

Fig. 8. a) Diagram eccentricity versus period (in days) for the $78 \mathrm{Ap}$ stars. The symbols are according to the type of Ap: $\triangle \mathrm{HgMn}$, $\Delta$ He weak, $\square \mathrm{SrCrEu}, \square \mathrm{Si}$. b) Diagram eccentricity versus period (in days) for G dwarf stars (Duquennoy \& Mayor 1991).

It is interesting to compare our $e / \log P$ diagram with that established by Debernardi (2002) for his large sample of Am stars. For these stars, the limit between circular and eccentric orbits is much steeper, many systems having $e \sim 0.7$ at $P=10 \mathrm{~d}$ only. The difference might be due to the wider mass distribution of the Bp-Ap stars (roughly 2 to $5 M_{\odot}$ ) compared to that of Am stars (1.5 to 2-2.5 $M_{\odot}$ ), $P_{\text {circ }}$ being different for each mass. One might also speculate that the lack of very eccentric and short periods is linked with the formation process of Ap stars, which for some reason (e.g. pseudo-synchronization in the PMS phase, leading to excessive equatorial velocities?) forbid this region.

Qualitatively, Ap stars behave roughly like normal Gdwarfs (Duquennoy \& Mayor 1991) in the eccentricity-period diagram. There is also a lack of low eccentricities at long periods (here for $\log P>2.0$ ), and the upper envelope is similar in both cases for $\log P>1.0$. One difference is the presence of moderate eccentricities for periods shorter than 10 days among Ap binaries, and another is the complete lack of very short orbital periods ( $P \leq 3$ days) among them, if one excepts HD 200405. The latter feature, already mentioned in the past (e.g. by GFH85) is especially striking because it does not occur for the Am binaries. The physical cause for it is probably that synchronization will take place rather early and force the components to rotate too fast to allow magnetic field and/or abundance anomalies to subsist. However, even an orbital period as short as one day will result in an equatorial velocity of only $152 \mathrm{~km} \mathrm{~s}^{-1}$ for a $3 R_{\odot}$ star, while single Bp or Ap stars rotating at this speed or even faster are known to exist (e.g. HD 60435, Bp SiMg, $P_{\text {rot }}=0.4755$ d; North et al. 1988). The detection of one system with $P_{\text {orb }}=1.635 \mathrm{~d}$ further 
complicates the problem, even though it remains an exceptional case as yet.

The shorter $P_{\text {circ }}$ value can be explained by the following effects:

- Ap stars are younger on average than $\mathrm{G}$ dwarfs, leaving less time for circularization;

- Ap stars have essentially radiative envelopes, on which tidal effects are less efficient: the circularization time increases much faster with the relative radius than for stars with convective envelopes: $t_{\text {circ }} \propto(a / R)^{21 / 2}$ instead of $t_{\text {circ }} \propto(a / R)^{8}$ (see e.g. Zahn 1977), $a$ being the semi-major axis of the relative orbit and $R$ the stellar radius;

- Therefore, it is essentially the secondary component which is responsible for the circularization of systems hosting an Ap star. Its radius being smaller than that of the Ap star, the circularization time is longer. Thus $=P_{\text {circ }}$ is shorter in these systems than in those hosting $\mathrm{G}$ dwarfs.

Our results confirm those of GFH85. Many stars of the literature have imprecise orbital elements, making a detailed analysis difficult. We have not enough CORAVEL orbits of Ap stars yet to make a more precise statistics.

\subsection{Mass function}

The mass function contains the unknown orbital inclination $i$. Therefore, neither the individual masses nor the mass ratio can be calculated from it. However the orbital inclination of Ap stars can be assumed to be randomly oriented on the sky. Thus, we can compare the observed cumulative distribution of the mass functions (for our 74 stars) with a simulated distribution (see Fig. 9), where we assume random orbit orientations. We approximated the observed relative distribution of Ap masses (North 1993) by the function given below, then multiplied it by Salpeter's law $\left(\mathcal{M}^{-2.35}\right)$ in order to obtain the simulated distribution of the primary masses $f_{\text {Ap }}$ :

$f_{\text {Ap }}=\left[0.3623+0.8764 \cdot \mathcal{M}+8.481 \cdot \mathrm{e}^{-(\mathcal{M}-3.5)^{2}}\right] \cdot \mathcal{M}^{-2.35}$

with a minimum mass of $1.5 \mathcal{M}_{\odot}$ and a maximum mass of $7 \mathcal{M}_{\odot}$.

In order to check the simulated distribution of masses of primaries, we estimated directly the masses of 60 primaries in systems having Geneva and uvby photometric measurements, as well as Hipparcos parallaxes. The estimation was done by interpolation through the evolutionary tracks of Schaller et al. (1992), using the method described by North (1998). The mass estimate was complicated by the binary nature of the stars under study. In the case of SB2 systems, the $V$ magnitude was increased by up to 0.75 , depending on the luminosity ratio, and the adopted effective temperature was either the photometric value or a published spectroscopic value. In the case of SB1 systems, only a fixed, statistical correction $\Delta V=0.2$ was brought to the $V$ magnitude, following North et al. (1997), which is valid for a magnitude difference of 1.7 between the components. The effective temperatures corresponding to the observed colours were increased by $3.5 \%$, to take into account a cooler companion with the same magnitude difference (assuming both companions are on the main sequence). In general $T_{\text {eff }}$
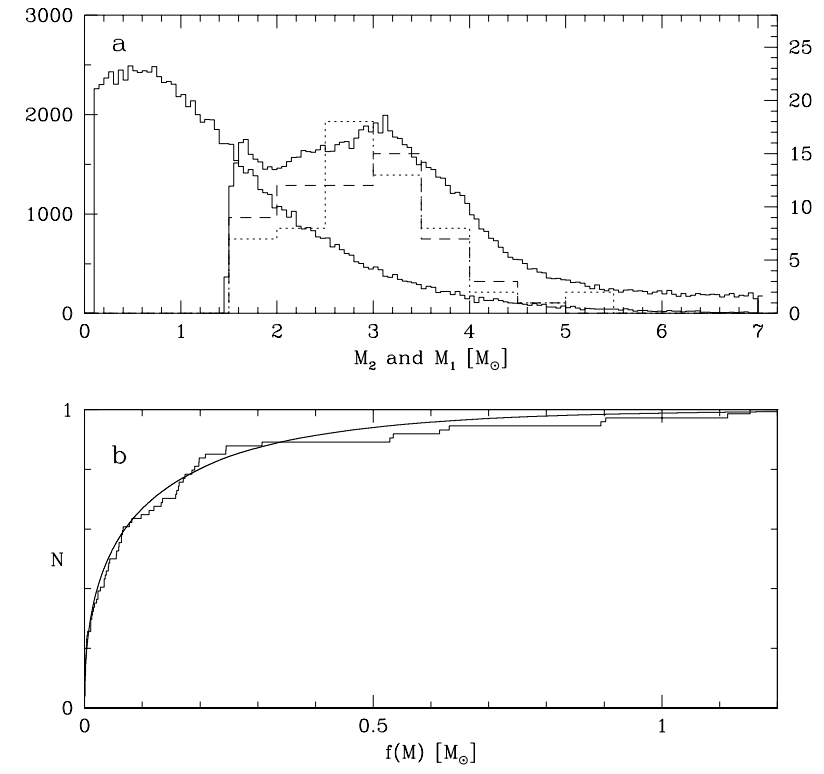

Fig. 9. a) Distributions of the masses of the secondary (left) and of the primary (right) used in simulation (100000 dots). The dotted and dashed lines represent the distributions of the primary masses of 60 stars of the sample respectively with and without applying the LutzKelker correction (1973). b) Observed and simulated cumulative distribution of the mass functions (including the He-weak and He-strong stars).

was obtained from Geneva photometry, using the reddeningfree $X$ and $Y$ parameters for stars hotter than about $9700 \mathrm{~K}$ and the dereddened (B2-G) index for cooler stars. The recipe used is described by North (1998). Existing $u v b y \beta$ photometry was used to check the validity of the $T_{\text {eff }}$ estimates and the $E(b-y)$ colour excess was used to estimate roughly the visual absorption through the relation $A_{\mathrm{V}}=4.3 E(b-y)$. The latter may be underestimated in some cases because the $b-y$ index of Ap stars is bluer than that of normal stars with same effective temperature, but only exceptionally by more than about $0.1 \mathrm{mag}$. Reddening maps by Lucke (1978) where used for consistency checks.

The resulting distribution of masses is represented, with an appropriate scaling, in Fig. 9a, together with the simulated distribution of primary masses. The agreement is reasonably good, justifying the expression used above for the distribution of primary masses.

For the distribution of secondary masses, we use the distribution of Duquennoy \& Mayor (1991) for the mass ratio $q=\frac{\mathcal{M}_{2}}{\mathcal{M}_{1}}$ of nearby G-dwarfs. We assume that the companions of the Ap stars are normal. The distribution of the mass ratio is a Gaussian:

$\xi(q) \propto \mathrm{e}^{-\frac{(q-\mu)^{2}}{2 \sigma_{q}^{2}}}$

with $\mu=0.23$ and $\sigma_{\mathrm{q}}=0.42$. Knowing the mass of the primary and the mass ratio, we can determine the mass of the secondary. We assume a minimal companion mass of $0.08 \mathcal{M}_{\odot}$. Using the test of Kolmogorov-Smirnov (Breiman 1973), we find a confidence-level for the observed and simulated cumulative distributions of $92 \%$. After elimination of the He-strong 
and He-weak stars, the KS test gives a confidence-level of $98 \%$. In other words, there is only a $2 \%$ probability that the observed distribution differs from the simulated one. This indicates that spectroscopic binaries with an Ap primary have the same distribution of mass ratios as binaries with normal components (Duquennoy \& Mayor 1991).

\subsection{Percentage of cool Ap stars as members of spectroscopic binary systems}

The sample used for estimating the binary percentage among cool Ap stars is only composed of the CORAVEL programme Ap stars described in the observation section, namely 119 stars. Including the stars HD 59435, HD 81009 and HD 137909 which have been published elsewhere and are not in Tables 1 and 2, the total number of programme stars is 122 . However, 6 of them have only one measurement, so they are not relevant here; in addition, about 3 stars have very large errors on their $R V$ values $\left(\sigma>3 \mathrm{~km} \mathrm{~s}^{-1}\right)$, so they are not reliable. Thus there are 113 objects on which statistics can be done. We chose, as variability criterion, the probability $P\left(\chi^{2}\right)$ that the variations of velocity are only due to the internal dispersion. A star will be considered as double or intrinsically variable if $P\left(\chi^{2}\right)$ is less than 0.01 (Duquennoy \& Mayor 1991). However, this test can not say anything about the nature of the variability.

For fast rotators, it is difficult to know whether the observed dispersion is just due to spots or betrays an orbital motion. Among the sample, 34 stars are assumed to be binaries, namely $30 \%$. Nevertheless, this figure has to be corrected for detection biases; we have attempted to estimate the rate of detection through a simulation. For this, a sample of 1000 double stars was created. The mass distributions explained above are used again. The orbital elements $T_{\mathrm{o}}, \omega$ and $i$ are selected from uniform distributions, while the eccentricity is fixed to zero for period less than 8 days and is distributed following a Gaussian with a mean equal to 0.31 and $\sigma=0.04$ (cases with negative eccentricity were dropped and replaced) for periods less than 1000 days and larger than 8 days, and following a distribution $f(e)=2 e$ for longer periods. The period is distributed according to a Gaussian distribution with a mean equal to $\overline{\log (P)}=4.8$ and $\sigma_{\overline{\log (P)}}=2.3$ (Duquennoy \& Mayor 1991), where $P$ is given in days. A cutoff at 2 and 5000 days was imposed. In a second step, the radial velocities of the created sample are computed at the epochs of observation of the real programme stars and with the real errors. Finally the $P\left(\chi^{2}\right)$ value of each star is computed. We obtain a simulated detection rate of $69 \%$.

After correction, we find a rate of binaries among Ap stars of about $43 \%$ in excellent agreement with the one determined by GFH 85 for the cool Ap stars of 44\%. Our sample is however more homogeneous and reliable.

\section{Conclusion}

We have determined a dozen of new spectroscopic orbits of Ap stars. Moreover, as shown in the Appendix, we have confirmed the 273-day period of HD 9996 and found a new value for the period of HD 216533 ( $P=1414.73$ days) in complete disagreement with the old values (16 days). We have computed the mass of both components of the Ap star 53 Cam, thanks to our homogeneous radial velocities which could be combined with the published speckle orbit. We have also shown that no significant apsidal motion has occurred in the HD 98088 system for the last 40 years.

The main result of this study is that statistically, the orbital parameters of Ap stars do not differ from those of normal stars, except for an almost complete lack of orbital periods shorter than 3 days. This cut-off is accompanied by a parallel lack of circular and low eccentricity orbits, the latter being due to the former. But in spite of this general rule, there is the interesting exception of HD 200405, an SB1 system with $P_{\text {rot }}=1.6$ days. This system would merit further investigation.

It is important to mention that the anomalous eccentricity distribution found by GFH85 is certainly not an independant fact, but is tightly linked with the lack of orbital periods shorter than 3 days. So short periods always correspond to circular orbits; therefore, removing them will result in an apparent excess of high eccentricities. There is nothing abnormal about the orbital parameters of binary systems hosting an Ap star, except for the lack of short periods.

The distribution of the mass ratios of Ap binaries is found to be compatible with the mass ratios of normal binaries with smaller masses (G-dwarfs). However, the sample of Ap stars with well-determined orbits is not sufficient to explore possible differences between the distributions of orbital parameters of each of the four categories of Ap stars on the one hand, and of the normal stars on the other hand.

\section{Appendix A: Results for individual systems}

\section{A.1. $H D 5550\left(=B D+65^{\circ} 115=\right.$ Renson 1470)}

This star had originally not been included in the CORAVEL sample because its photometric effective temperature exceeds $10000 \mathrm{~K}$. On the other hand, it was included in the sample measured with ELODIE for the survey of surface magnetic fields, which allowed us to discover its SB2 nature. The CORAVEL data show that it is a short period binary, and allow us to obtain the orbital parameters. The SB2 nature of this star is not visible with the CORAVEL instrument while it is clear with ELODIE. In addition to the better resolution of ELODIE, this might be due to the fact that CORAVEL covers only the blue wavelength range, while ELODIE is rather sensitive to the red. The star visible with CORAVEL is the less massive one (see Fig. A.1), so if significantly redder, the invisible companion could only be a red giant. This is forbidden by the short orbital period: the shortest orbital period for systems hosting a red giant is about 40 days (Mermilliod, private communication). Therefore, the more massive component must be also the hotter one, and its visibility with ELODIE is probably linked with the mask used for the correlation, which had been specifically defined for Ap stars, while the CORAVEL mask was designed from the spectrum of Arcturus. This raises the interesting possibility that both companions might be Ap stars (or possibly Am), since otherwise their metallicity would not have been sufficient to yield correlation dips. Another favorable circumstance is the rather low inclination of the system, since for an assumed mass $M_{1}=2.5 M_{\odot}$ (for the most massive component), the inclination 
$i=20.5^{\circ}$ only, so that the projected rotational velocity is only 35 percent of the equatorial value. The CORAVEL data then do not allow to obtain the radial-velocity curve of the primary; on the other hand, they yield the systemic velocity which, together with the two ELODIE spectra, allow a fairly good estimate of the mass ratio (see Table A.1).

This star is remarkable, because it is only the fifth SB2 system known among magnetic Ap stars, after HD 55719 (Bonsack 1976), HD 98088 (Abt et al. 1968; Wolff 1974), HD 59435 (Wade et al. 1996) and HD 174016-7 (Ginestet et al. 1999); HD 59435 had also been studied in the course of this survey, while a sixth case (HD 22128, see below) was discovered with ELODIE. Although HgMn stars are frequently seen in SB2 systems, this is exceptional among $\mathrm{Si}$ and $\mathrm{SrCrEu}$ stars.

\section{A.2. $H D 9996\left(=B D+44^{\circ} 341=\right.$ Renson 2470$)$}

The duplicity of this B9 CrEuSi (Osawa 1965) star has first been detected by Preston \& Wolff (1970) who found an orbital period of 273 days. They did not attempt to determine the orbital elements because of the poverty of the data. Scholz (1978) tried to determine the orbital elements, but the shape of the velocity curve in the vicinity of the maximum remained illdefined. The 43 CORAVEL measurements (Table 1) confirm the 273-day period (see Fig. A.1). Thanks to the precision and homogeneity of the data, our velocity curve is more precise than the one based on the data gathered by Preston \& Wolff (1970) and Scholz (1978), so that a satisfactory determination of the orbital elements is possible.

The rather large rms scatter of the residuals $\left(1.24 \mathrm{~km} \mathrm{~s}^{-1}\right)$ is due to the small depth of the correlation dip ( 3 percent). Rotation is not important, the $v \sin i$ value of the visible component is very small $\left(<2 \mathrm{~km} \mathrm{~s}^{-1}\right.$, see Tables 2 and A.4) and has no effect on the correlation-peak width; this is confirmed by the very long rotational period of the primary $(\sim 21$ years, Rice 1988), so that even highly contrasted abundance spots could not distort the radial-velocity curve.

\section{A.3. $H D 12288\left(=B D+68^{\circ} 144=\right.$ Renson 3130)}

This star was classified A2 CrSi by Osawa (1965). Its rotational period, known from its magnetic variability, is 34.79 days (Mathys et al. 1997). Thirty-one observations were obtained over an interval of 5949 days (Table 1), which represents about 4 orbital periods $\left(P_{\text {orb }}=1547 \mathrm{~d}\right)$. The radial-velocity curve is shown in Fig. A.1. The projected rotational velocity estimated from the width of the autocorrelation dip is moderate but significant (Tables 2, A.4), but should not be considered as reliable because the effect of the magnetic field is not taken into account in this estimate. Since the Zeeman effect will always widen the dip, the $v \sin i$ values listed in Tables 2 and A.4 must be considered as upper limits to the true projected rotational velocity. If considered with this caution in mind, they are very useful.

\section{A.4. $H D 22128(=B D-070624=$ Renson 5560)}

This A7 SrEuMn star (Renson 1991) was found to be an SB2 system during the survey for magnetic fields carried out with ELODIE. We do not have Geneva photometry for that star, but only Strömgren photometry ${ }^{3}$ given by Olsen $(1983,1994)$. The average physical parameters obtained from the $u v b y \beta$ values compiled by Mermilliod et al. (1997) (assuming both components are identical) and using the calibration of Moon \& Dworetsky (1985) are listed in Table A.3. From the physical parameters we obtain a typical mass $\mathcal{M}=1.99 \pm 0.17 \mathcal{M}_{\odot}$, according to the models of Schaller et al. (1992). The inclination angle $i$ may be estimated close to $48^{\circ}$.

Notice, in Fig. A.1, that the radial-velocity curve is very close to a circular orbit $(e=0.0145 \pm 0.0116)$. Therefore, the test of Lucy \& Sweeney (1971) was applied in order to see whether this small eccentricity is significant or not. The probability $\mathrm{p}$ is equal to 0.69 in our case, which is much greater than the limit of 0.05 determined by Lucy \& Sweeney. Thus this eccentricity of 0.0145 is not significant and is fixed to zero.

\section{A.5. $H D 40711$ (= BD +10 $973=$ Renson 10880)}

Bidelman \& McConnell (1973) classified this object Ap SrCrEu. Geneva photometry clearly confirms the peculiarity with $\Delta(V 1-G)=0.020$ (the photometric data in the Geneva system are collected in the General Catalogue - Rufener 1988 - and its up-to-date database - Burki 2002). The radial velocities are represented in Fig. A.1. The periastron was observed again only recently, which allowed a precise estimate of the orbital period. The eccentricity is high and relatively well defined, though the exact shape of the $R V$ curve in the vicinity of the periastron remains unknown because of the 7-weeks gap in the observations. The depth of the dip varies, while its width only shows rather marginal changes.

\section{A.6. $H D 54908\left(=B D-01^{\circ} 1579=\right.$ Renson 15000)}

HD 54908 is a poorly studied Ap star classified A0 Si by Bidelman \& McConnell (1973). In spite of a large $v \sin i=$ $53.6 \pm 5.34 \mathrm{~km} \mathrm{~s}^{-1}$, the variation of the radial velocity is too large to be caused by spots and rotation $(K=27.47 \pm$ $1.14 \mathrm{~km} \mathrm{~s}^{-1}$ ). However we can see the effect of a large rotational velocity on the scatter of the residuals. The twentyone observations were obtained over an interval of 4084 days. The shape of the radial-velocity curve (Fig. A.1) is not very well defined in the vicinity of the minimum, but the period of 17.92 days is quite well determined.

\section{A.7. HD $56495(=B D-0701851=$ Renson 15430 $)$}

This star was classified A3p Sr by Bertaud (1959), which motivated its inclusion in our sample, but Bertaud \& Floquet (1967) classified it A2-F2 (Am). Its classification remains ambiguous, and it would be interesting to know its $\Delta a$ index in Maitzen's (1976) photometry. Its peculiarity index in Geneva photometry

${ }^{3} V=7.595, b-y=0.228, m_{1}=0.220, c_{1}=0.672$. 

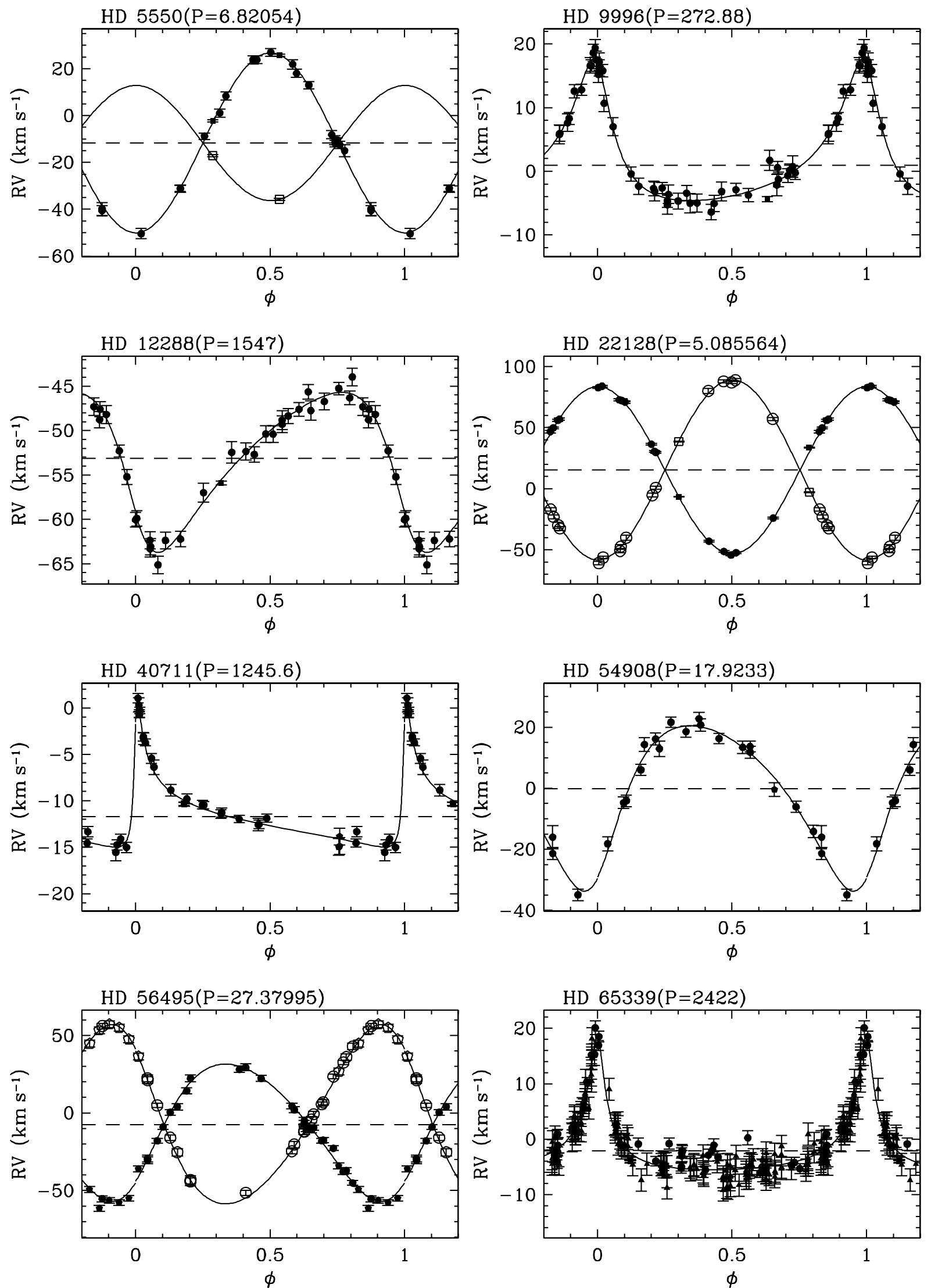

Fig. A.1. Phase-folded radial-velocity curves of the first eight binaries listed in Table 1. Full dots: CORAVEL observations; triangles: ELODIE observations. 
Table A.1. Orbital parameters of the binaries. For each component, the second line gives the estimated standard deviations of the parameters.

\begin{tabular}{|c|c|c|c|c|c|c|c|c|c|c|}
\hline Star name & $\begin{array}{c}P \\
\text { (days) }\end{array}$ & $\begin{array}{c}T_{\circ}(\mathrm{HJD} \\
-2400000) \\
\end{array}$ & $e$ & $\begin{array}{c}V_{\circ} \\
\left(\mathrm{km} \mathrm{s}^{-1}\right)\end{array}$ & $\begin{array}{l}\omega_{1} \\
\left({ }^{\circ}\right)\end{array}$ & $\begin{array}{c}K_{1,2} \\
\left(\mathrm{~km} \mathrm{~s}^{-1}\right)\end{array}$ & $\begin{array}{c}\mathcal{M}_{1,2} \sin ^{3} i \\
f_{1}(\mathcal{M})\end{array}$ & $\begin{array}{c}a_{1,2} \sin i \\
\left(10^{6} \mathrm{~km}\right)\end{array}$ & $N$ & $\begin{array}{c}(\mathrm{O}-\mathrm{C}) \\
\left(\mathrm{km} \mathrm{s}^{-1}\right)\end{array}$ \\
\hline \multirow[t]{4}{*}{ HD 5550} & 6.82054 & 50988.70 & 0.00 & -11.70 & - & 24.60 & 0.1081 & 2.307 & 2 & 1.13 \\
\hline & 0.00020 & 0.011 & fixed & 0.28 & - & 0.82 & 0.0045 & 0.077 & & \\
\hline & & & & & - & 38.43 & 0.0692 & 3.605 & 22 & \\
\hline & & & & & - & 0.46 & 0.0036 & 0.043 & & \\
\hline \multirow[t]{2}{*}{ HD 9996} & 272.88 & 44492.34 & 0.532 & 0.97 & 20.17 & 11.12 & 0.0237 & 35.34 & 43 & 1.33 \\
\hline & 0.20 & 2.24 & 0.023 & 0.22 & 3.35 & 0.29 & 0.0022 & 1.11 & & \\
\hline \multirow{2}{*}{ HD 12288} & 1546.99 & 44480.5 & 0.337 & -53.15 & 120.84 & 9.01 & 0.0982 & 180.5 & 31 & 0.84 \\
\hline & 7.29 & 15.8 & 0.024 & 0.16 & 5.49 & 0.26 & 0.0089 & 5.5 & & \\
\hline \multirow[t]{4}{*}{ HD 22128} & 5.085564 & 50116.7656 & 0.00 & 15.30 & - & 68.40 & 0.786 & 4.784 & 20 & 1.25 \\
\hline & 0.000070 & 0.0043 & fixed & 0.21 & - & 0.37 & 0.012 & 0.026 & & \\
\hline & & & & & - & 73.69 & 0.729 & 5.153 & 18 & \\
\hline & & & & & - & 0.55 & 0.010 & 0.038 & & \\
\hline \multirow[t]{2}{*}{ HD 40711} & 1245.6 & 49591.7 & 0.834 & -11.69 & 314.3 & 7.88 & 0.0106 & 74.5 & 31 & 0.51 \\
\hline & 4.4 & 6.3 & 0.013 & 0.12 & 2.1 & 0.46 & 0.0022 & 5.1 & & \\
\hline \multirow[t]{2}{*}{ HD 54908} & 17.9233 & 46469.96 & 0.286 & -0.15 & 213.45 & 27.09 & 0.0326 & 6.40 & 21 & 2.58 \\
\hline & 0.0017 & 0.36 & 0.034 & 0.61 & 6.47 & 1.19 & 0.0044 & 0.29 & & \\
\hline \multirow[t]{4}{*}{ HD 56495} & 27.37995 & 48978.40 & 0.1651 & -7.57 & 224.7 & 44.30 & 1.641 & 16.45 & 32 & 2.45 \\
\hline & 0.00080 & 0.23 & 0.0097 & 0.35 & 3.2 & 0.74 & 0.055 & 0.27 & & \\
\hline & & & & & 44.7 & 57.75 & 1.259 & 21.44 & 28 & \\
\hline & & & & & 3.2 & 0.81 & 0.044 & 0.30 & & \\
\hline \multirow{2}{*}{$\begin{array}{r}\text { HD } 65339 \\
\text { (RV only) }\end{array}$} & 2422.04 & 27723.6 & 0.718 & -2.10 & 5.22 & 12.08 & 0.149 & 280.0 & 181 & 1.72 \\
\hline & 2.42 & 14.3 & 0.012 & 0.14 & 1.64 & 0.45 & 0.019 & 11.7 & & \\
\hline \multirow[t]{2}{*}{ HD 73709} & 7.220263 & 49996.5352 & 0.00 & 36.51 & - & 30.84 & 0.02200 & 3.062 & 45 & 0.89 \\
\hline & 0.000017 & 0.0093 & fixed & 0.13 & - & 0.19 & 0.00041 & 0.019 & & \\
\hline \multirow[t]{4}{*}{ HD 98088} & 5.905111 & 34401.387 & 0.1796 & -8.45 & 314.46 & 73.29 & 1.733 & 5.854 & 88 & 2.34 \\
\hline & 0.000004 & 0.023 & 0.0039 & 0.23 & 1.44 & 0.36 & 0.030 & 0.029 & & \\
\hline & & & & & 134.46 & 99.46 & 1.277 & 7.940 & 19 & \\
\hline & & & & & 1.44 & 0.90 & 0.020 & 0.070 & & \\
\hline \multirow[t]{2}{*}{ HD 105680} & 70.0795 & 45991.19 & 0.3798 & -5.13 & 192.6 & 30.75 & 0.1676 & 27.42 & 42 & 0.76 \\
\hline & 0.0087 & 0.38 & 0.0055 & 0.13 & 1.1 & 0.20 & 0.0034 & 0.19 & & \\
\hline \multirow[t]{2}{*}{ HD 138426} & 11.34474 & 48690.398 & 0.512 & -14.63 & 121.37 & 44.02 & 0.0636 & 5.90 & 20 & 2.04 \\
\hline & 0.00029 & 0.052 & 0.020 & 0.52 & 2.81 & 1.46 & 0.0068 & 0.21 & & \\
\hline \multirow[t]{2}{*}{ HD 184471} & 429.17 & 46857.06 & 0.2017 & -26.16 & 86.99 & 15.59 & 0.1585 & 90.09 & 36 & 0.51 \\
\hline & 0.42 & 3.01 & 0.0081 & 0.12 & 2.81 & 0.15 & 0.0045 & 0.86 & & \\
\hline \multirow[t]{2}{*}{ HD 188854} & 8.480322 & 46394.223 & 0.2262 & -29.61 & 23.84 & 41.76 & 0.05926 & 4.743 & 34 & 0.40 \\
\hline & 0.000025 & 0.014 & 0.0024 & 0.07 & 0.64 & 0.10 & 0.00045 & 0.012 & & \\
\hline \multirow[t]{2}{*}{ HD 200405} & 1.635255 & 46999.9766 & 0.00 & -0.974 & 0.00 & 8.44 & 0.0001021 & 0.190 & 34 & 0.63 \\
\hline & 0.000006 & 0.0058 & fixed & 0.12 & fixed & 0.18 & 0.0000064 & 0.004 & & \\
\hline \multirow[t]{2}{*}{ HD 216533} & 1413.1 & 43752.5 & 0.437 & -4.05 & 182.8 & 5.04 & 0.0137 & 88.1 & 48 & 0.71 \\
\hline & 4.6 & 17.4 & 0.026 & 0.11 & 4.9 & 0.22 & 0.0019 & 4.0 & & \\
\hline
\end{tabular}

is $\Delta(V 1-G)=-0.006$ only, which is typical of normal stars, but the efficiency of this index is known to be low for such cool Ap stars. This is an excentric SB2 system, whose inclination angle $i$ remains unknown. We secured 60 points (Fig. A.1) and obtained the orbital elements listed in Table A.1.

A rough estimate of the inclination angle $i$ and of the masses of the components can be done using uvby $\beta$ photometry ${ }^{4}$ and the calibration by Moon \& Dworetsky (1985). The physical parameters obtained are listed in Table A.3.

\footnotetext{
${ }^{4} V=7.68, b-y=0.194, m_{1}=0.214, c_{1}=0.679, \beta=2.739$, by Cameron (1966).
}

Combining these results with the models of Schaller et al. (1992), one finds an approximate mass $\mathcal{M}=1.80 \pm 0.09 \mathcal{M}_{\odot}$ and an inclination $i$ close to $75^{\circ}$.

\section{A.8. $H D 65339\left(=53 \mathrm{Cam}=\mathrm{BD}+60^{\circ} 1105=\right.$ Renson 17910)}

53 CAM is a very well studied A3 SrEuCr star (Osawa 1965). It is known as a binary by both spectroscopy and speckle interferometry. The speckle orbit was published by Hartkopf et al. (1996) and a radial-velocity curve was published by Scholz \& Lehmann (1988). Combining our 46 measurements (Table 1) 
Table A.2. Orbital parameters of HD 191654, assuming that the $R V$ variations of this star is caused by orbital motion rather than by rotating abundance patches on its surface.

\begin{tabular}{rrrrrrrrrrr}
\hline \hline Star name & $\begin{array}{c}P \\
\text { (days) }\end{array}$ & $\begin{array}{r}T_{\circ}(\mathrm{HJD} \\
-2400000)\end{array}$ & $e$ & $\begin{array}{c}V_{\circ} \\
\left(\mathrm{km} \mathrm{s}^{-1}\right)\end{array}$ & $\begin{array}{c}\omega_{1} \\
\left(^{\circ}\right)\end{array}$ & $\begin{array}{c}K_{1,2} \\
\left(\mathrm{~km} \mathrm{~s}^{-1}\right)\end{array}$ & $\begin{array}{c}\mathcal{M}_{1,2} \sin ^{3} i \\
f_{1}(\mathcal{M})\end{array}$ & $\begin{array}{c}a_{1,2} \sin i \\
10^{6}\end{array}$ & $\begin{array}{r}N \\
\mathrm{~km}\end{array}$ & $\begin{array}{r}(\mathrm{O}-\mathrm{C}) \\
\mathrm{km} \mathrm{s}^{-1}\end{array}$ \\
\hline HD 191654 & 2121. & 48692. & 0.48 & -15.72 & 88. & 2.11 & 0.00140 & 54.0 & 27 & 0.91 \\
& 27. & 50. & 0.10 & 0.23 & 17. & 0.24 & 0.00055 & 7.1 & \\
\hline
\end{tabular}

Table A.3. Physical parameters of HD 22128 and HD 56495 according to their colours in the $u v b y \beta$ or Geneva photometric system.

\begin{tabular}{c|ccccccccc}
\hline \hline Star & Photometry & $T_{\text {eff }}[K]$ & $\log g[\mathrm{cgs}]$ & {$[\mathrm{M} / \mathrm{H}]$} & $\log \left(L / L_{\odot}\right)$ & $R / R_{\odot}$ & $M_{v}$ & $M_{\text {bol }}$ & $\Delta m_{0}$ \\
\hline HD 22128 & $u v b y \beta$ & 6900 & 3.65 & 0.57 & 0.95 & 2.10 & 2.29 & 2.26 & -0.052 \\
\hline HD 56495 & $u v b y \beta$ & 7179 & 4.00 & 0.42 & 0.77 & 1.58 & 2.77 & 2.72 & -0.032 \\
& Geneva & $7044 \pm 56$ & $4.26 \pm 0.09$ & $0.26 \pm 0.08$ & & & & & \\
\hline
\end{tabular}

Table A.4. Physical parameters of the binaries according to their colours in the Geneva photometric system (or in the uvby $\beta$ system for HD 22128); in the case of HD 5550, we have adopted the $T_{\text {eff }}$ value estimated from the $\mathrm{H}_{\alpha}$ profile observed with ELODIE near conjonction. The reddenings $E(B 2-G)$ labeled with an asterisk are determined using the maps of Lucke (1978). $v \sin i$ is obtained by a calibration of the CORAVEL correlation-dip width (Benz \& Mayor 1984). The resulting $v \sin i$ is slightly less reliable than for $\mathrm{F}$ and cooler stars which were used for the calibration, because their effective temperature is larger and the corresponding dependance has to be extrapolated. However, the main source of uncertainty is due to disregarding the magnetic field, which implies an overestimate of $v \sin i$. The longitudinal magnetic field is taken from Babcock (1958) or more recent references. ${ }^{*}$ Value given by Debernardi et al. (2000).

\begin{tabular}{rrrrrr}
\hline \hline HD & $\begin{array}{r}v \sin i \text { upper limits } \\
\left(\mathrm{km} \mathrm{s}^{-1}\right)\end{array}$ & $m_{V}$ & $\begin{array}{c}H_{z} \\
(\mathrm{KG})\end{array}$ & $\begin{array}{c}T_{\text {eff }} \\
(\mathrm{K})\end{array}$ & $E(B 2-G)$ \\
\hline 5550 & $6.5 \pm 1.3$ & 5.967 & - & 11000 & 0.046 \\
9996 & 2.0 & 6.379 & -1.2 to 0.3 & 9700 & 0.017 \\
12288 & $12.5 \pm 0.4$ & 7.748 & -1.2 to -0.2 & 9378 & 0.175 \\
$22128 \mathrm{~A}$ & $15.9 \pm 0.3$ & 7.595 & - & 7000 & $0^{*}$ \\
$22128 \mathrm{~B}$ & $16.3 \pm 0.7$ & & - & & \\
40711 & 2.0 & 8.581 & - & 9328 & $0.192^{*}$ \\
54908 & $55.2 \pm 5.5$ & 7.968 & - & 7483 & 0.031 \\
$56495 \mathrm{~A}$ & $25.3 \pm 2.5$ & 7.654 & 0.21 to 0.57 & 7044 & $0^{*}$ \\
$56495 \mathrm{~B}$ & $12.5 \pm 2.1$ & 7.654 & - & & \\
65339 & $19.4 \pm 0.6$ & 6.031 & -5.4 to 4.2 & 8250 & 0.012 \\
73709 & $17.3 \pm 0.3 *$ & 7.687 & - & 7831 & $0 *$ \\
$98088 \mathrm{~A}$ & $21.1 \pm 2.1$ & 6.42 & 0.48 to 0.94 & 8043 & $0^{*}$ \\
$98088 \mathrm{~B}$ & $15.8 \pm 1.9$ & 7.62 & & 7532 & $0^{*}$ \\
105680 & $14.1 \pm 0.2$ & 8.060 & - & 7154 & $0^{*}$ \\
138426 & 2.0 & 8.546 & - & 8694 & 0.142 \\
184471 & 2.0 & 8.980 & - & 8114 & 0.116 \\
188854 & $9.3 \pm 0.2$ & 7.634 & - & 7005 & $0.069^{*}$ \\
200405 & $9.6 \pm 0.4$ & 8.908 & - & 9624 & 0.101 \\
216533 & $5.7 \pm 0.3$ & 7.907 & -0.7 to 0.1 & 9000 & 0.120 \\
\hline
\end{tabular}

with those published by Scholz \& Lehmann (1988), we determine the orbital parameters listed in Table A.1. The scatter of the residuals of Scholz's measurements are similar to those of CORAVEL observations alone, which appears surprising at first sight. Examining the depth and width of the correlation dip as a function of the rotational phase $(P=8.0267$ days $)$, one clearly sees a significant variation of both quantities (see Fig. 4). The residuals around the fitted $R V$ curve also show a variation, which is related, therefore, to the spots associated with a non-negligible $v \sin i .53 \mathrm{Cam}$ is then a nice example of an object displaying two variations simultaneously, one due to rotation (with an amplitude of up to $7 \mathrm{~km} \mathrm{~s}^{-1}$ peak-to-peak) and the other due to a binary companion.
Thanks to a code made available by T. Forveille and developed in Grenoble, we have fitted simultaneously the radial velocities, the speckle measurements and the Hipparcos parallax $(\pi=10.16 \pm 0.77)$, leaving not only $K_{1}$ but also $K_{2}$ as an adjustable parameter in spite of the lack of $R V$ data for the companion. The speckle measurements retained for the fit are given in Table A.5, while the results are shown in Table A.6. The "visual" orbit is shown in Fig. A.3.

This is the first time that such a solution is attempted for this system. The results are surprising, in that both companions appear to have the same mass, contrary to what Scholz \& Lehman (1988) had found $\left(2.5 \mathcal{M}_{\odot}\right.$ and $1.6 \mathcal{M}_{\odot}$ for the primary and the secondary respectively) by combining the 

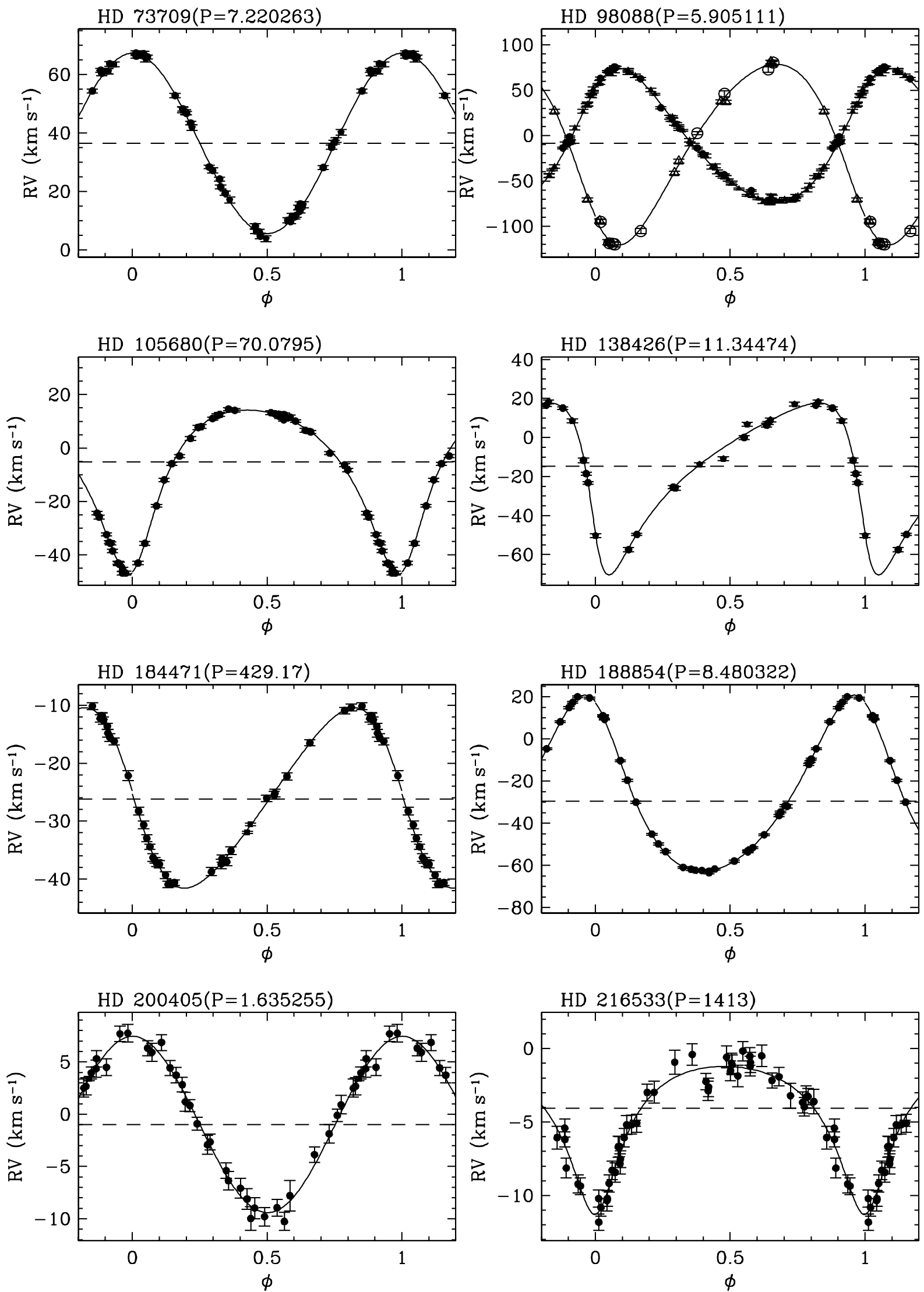

Fig. A.2. Phase-folded radial-velocity curves of the last eight binaries listed in Table 1. Full dots: CORAVEL observations; triangles: ELODIE observations. 
Table A.5. Speckle observations of HD 65339 used for the simultaneous fit on the $R V$, speckle and parallax data. Since the errors are not given by the authors, an error of 0.01 arcsec and of $2^{\circ}$ has been assumed on the separation and position angle respectively.

\begin{tabular}{|c|c|c|c|}
\hline $\begin{array}{r}\text { Epoch } \\
\text { (frac. year) }\end{array}$ & $\begin{array}{r}\rho \\
(\operatorname{arcsec})\end{array}$ & $\begin{array}{r}\theta \\
\left(^{\circ}\right)\end{array}$ & Source \\
\hline 1980.1561 & 0.044 & 336.4 & McAlister et al. (1983) \\
\hline 1984.0526 & 0.093 & 299.6 & McAlister et al. (1987) \\
\hline 1984.8463 & 0.091 & 307.3 & Balega $^{2}$ (1987) \\
\hline 1985.1830 & 0.091 & 306.3 & Balega $^{2}(1987)$ \\
\hline 1985.1858 & 0.088 & 308.7 & Balega $^{2}(1987)$ \\
\hline 1986.7039 & 0.045 & 328.6 & Balega et al. (1989) \\
\hline 1986.8894 & 0.0339 & 332.44 & Hartkopf et al. (1996) \\
\hline 1989.2267 & 0.063 & 283.1 & McAlister et al. (1990) \\
\hline 1990.2755 & 0.089 & 293.1 & Hartkopf et al. (1992) \\
\hline 1991.3265 & 0.086 & 303.8 & Hartkopf et al. (1994) \\
\hline 1991.8943 & 0.085 & 306.7 & Hartkopf et al. (1994) \\
\hline 1992.3124 & 0.080 & 310.7 & Hartkopf et al. (1994) \\
\hline
\end{tabular}

separate $R V$ and speckle orbits (they used a photometric mass for the primary, since there was no good parallax value at the time). On the other hand, they are compatible with the small $\Delta m$ required for speckle observations. They also differ from the mass estimate done by Martin \& Mignard (1998) on the basis of Hipparcos results, which has a large error, however. The uncertainty is very large and could be substantially reduced if the spectrum of the secondary could be observed. We could not see it on our ELODIE spectra, but this is not surprising since they were taken when both companions had almost the same radial velocity.

\section{A.9. $H D 73709\left(=B D+20^{\circ} 2165=\right.$ Renson $20510=$ Praesepe KW 279)}

HD 73709 was classified A2-A5-F0 (Am) by Gray \& Garrison (1989), but was found photometrically Ap by Maitzen \& Pavlovski (1987) according to the $\Delta a$ index $(\Delta a=0.018)$. The Geneva peculiarity index gives an ambiguous answer: $\Delta(V 1-G)=0.001$ is a few thousands of magnitude larger than the average of normal stars, but is not conspicuous. It has been put lately in our programme because of its photometric peculiarity, first for magnetic field measurements, second for radial-velocity monitoring.

Two ELODIE data were taken in the course of the survey for magnetic fields, while a third one has kindly been obtained for us by Mr. Dominique Naef (Geneva Observatory) during a planet-search programme.

HD 73709 is extremely interesting because of its reliable Am classification and positive $\Delta a$ : it was generally accepted that Am stars never show enhanced $\Delta a$ values (Maitzen 1976; Maitzen et al. 1998) which are characteristic of magnetic Ap stars only. Conversely, large-scale magnetic fields are generally not found in Am stars, with the probable exception of the hot Am star $o$ Peg (Mathys 1988; Mathys \& Lanz 1990). The three spectra taken with the ELODIE spectrograph consistently show a surface magnetic field of about $7.5 \mathrm{kG}$ which seems very significant, in spite of a relatively large projected rotational velocity $v \sin i=16 \mathrm{~km} \mathrm{~s}^{-1}$ (Babel \& North, in preparation).

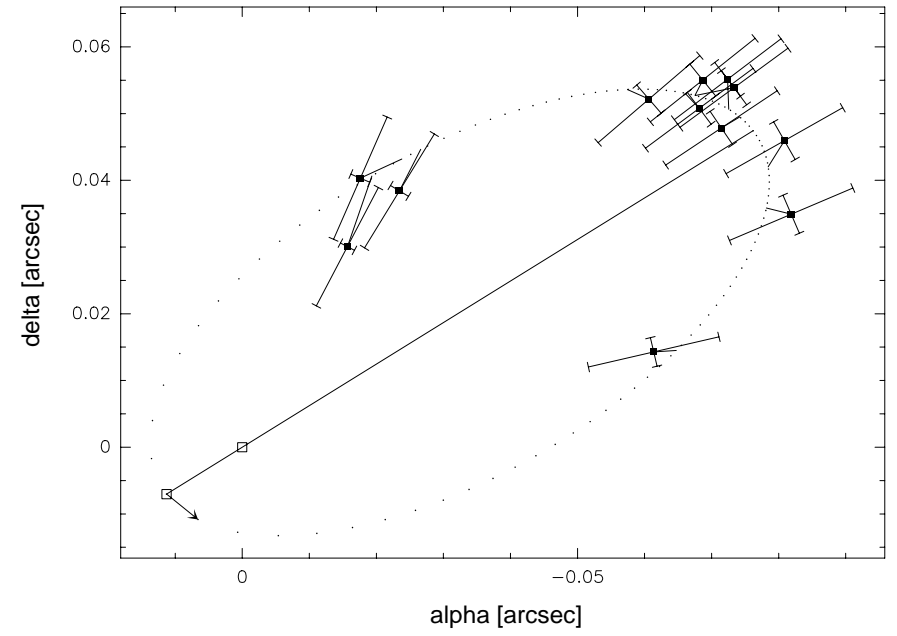

Fig. A.3. Orbit of the system HD 65339 projected on the sky, as fitted to both radial velocities and speckle measurements simultaneously. The axes are labeled in arcseconds. The error bars have been chosen in an arbitrary way because they are not given by the authors.

The orbit of this star was published by Debernardi et al. (2000). However, we have 12 additional data, so we have redetermined the orbit using both published and new data (note that the data published by Debernardi et al. have not been put into the ELODIE $R V$ system (Udry et al. 1999), so that the $R V$ values used here are very slightly different from those published by these authors). The orbit is slightly improved.

\section{A.10. $H D$ 98088A $\left(=B D-6^{\circ} 3344 A=\right.$ Renson 28310)}

This is a well-known SB2 binary hosting a magnetic Ap star of the type $\mathrm{SrCr}$ according to Osawa (1965). Its binary nature has been discovered by Abt (1953), who saw it only as an SB1, and the complete orbital solution of the SB2 system was given by Abt et al. (1968). These authors have shown that the spectral variations have the same period as the orbital one and that the system must, therefore, be synchronized. According to them, the spectral type of the primary is $\mathrm{A} 3 \mathrm{Vp}$ while that of the secondary is A8V. In spite of the binary nature of this star, the Geneva photometric system "sees" its peculiarity, with $\Delta(V 1-G)=0.013$, and Maitzen's (1976) photometry is even more efficient, with $\Delta a=0.035$. Therefore, the primary is a rather extreme Ap star.

A very interesting feature of HD 98088 is that, in spite of its relatively short orbital period, it has a significant eccentricity, so that one may expect an apsidal motion to take place. According to Wolff (1974), only marginal evidence for such a motion could be found over a time base of 20 years, and new observations should be done 20 to 30 years later to settle the question, the expected period of the apsidal motion being 500 to 700 years. Because of this expectation, we reobserved the system with CORAVEL in the spring of 1998. Bad weather prevented us to obtain a dense coverage of all phases, but 17 observations of the primary and 8 of the secondary could be done. The period could be refined to

$P=5.905111 \pm 0.000004$ days. 
Table A.6. Orbital parameters of HD 65339 obtained with a simultaneous fit on the $R V$, speckle and parallax data.

\begin{tabular}{|l|r|r|r|r|r|r|r|r|r|r|r|}
\hline Star name & $\begin{array}{c}P \\
\text { (days) }\end{array}$ & $\begin{array}{c}T_{\circ}(\mathrm{HJD} \\
-2400000)\end{array}$ & $e$ & $\begin{array}{c}V_{\circ} \\
\left(\mathrm{km} \mathrm{s}^{-1}\right)\end{array}$ & $\begin{array}{c}\Omega_{1} \\
\left(^{\circ}\right)\end{array}$ & $\begin{array}{c}\omega_{1} \\
\left({ }^{\circ}\right)\end{array}$ & $\begin{array}{c}i \\
\left({ }^{\circ}\right)\end{array}$ & $\begin{array}{c}K_{1,2} \\
\left(\mathrm{~km} \mathrm{~s}^{-1}\right)\end{array}$ & $\begin{array}{c}\mathcal{M}_{1,2} \\
\left(\mathcal{M}_{\odot}\right)\end{array}$ & $\begin{array}{c}a_{1,2} \\
\left(10^{6} \mathrm{~km}\right)\end{array}$ & $\begin{array}{c}\pi \\
(\mathrm{mas})\end{array}$ \\
\hline HD 65339 & 2418.9 & 27738.8 & 0.742 & -1.94 & 116.80 & 7.30 & 134.3 & 12.33 & 1.49 & 275.1 & 10.2 \\
$(R V$ & 2.41 & 15.6 & 0.013 & 0.13 & 1.31 & 1.37 & 4.4 & 0.41 & 0.66 & & 1.0 \\
+speckle $)$ & & & & & & & & & & \\
& & & & & & & & 12.13 & 1.52 & 270.5 & \\
& & & & & & & & 3.25 & 0.33 & & \\
\hline
\end{tabular}

Table A.7. Physical parameters of both components of the spectroscopic system HD 98088A, inferred from photometric temperatures and Hipparcos parallax.

\begin{tabular}{lcc}
\hline \hline Parameter & primary & secondary \\
\hline$M_{V}$ & 0.87 & 2.07 \\
$\log \left(T_{\text {eff }}\right)$ & $3.905 \pm 0.016$ & $3.877 \pm 0.017$ \\
$\log \left(L / L_{\odot}\right)$ & $1.60 \pm 0.09$ & $1.10 \pm 0.09$ \\
$M\left(M_{\odot}\right)$ & $2.261 \pm 0.093$ & $1.755 \pm 0.085$ \\
$R\left(R_{\odot}\right)$ & $3.27 \pm 0.43$ & $2.10 \pm 0.28$ \\
$\log g(\mathrm{cgs})$ & $3.76 \pm 0.10$ & $4.04 \pm 0.11$ \\
\hline$R \sin i=\frac{P \cdot v \sin i}{50.6}\left(R_{\odot}\right)$ & $2.46 \pm 0.25$ & $1.84 \pm 0.22$ \\
$R=R \sin i / \sin \left(66^{\circ}\right)$ & $2.70 \pm 0.27$ & $2.02 \pm 0.24$ \\
$d(\mathrm{pc})$ & \multicolumn{2}{c}{$129 \pm 13$} \\
\hline
\end{tabular}

The $\omega$ angle has not changed in a significant way since about 30 years, since we find $\omega_{1}=314.25 \pm 3.66^{\circ}$, while the combined literature data for the epochs 1953-1973 give $\omega_{1}=$ $314.41 \pm 1.09^{\circ}$ according to Wolff (1974). We verified this result with our code, which gives practically the same value but a larger uncertainty $\left(\omega_{1}=314.47 \pm 1.60^{\circ}\right.$, for the primary $R V$ curve alone). If the period of the apsidal motion was 700 years as suggested by Wolff (1974), then the argument of the periastron should have changed by $18^{\circ}$ in 35 years, so we should have found $\omega_{1} \sim 332.5^{\circ}$. This is five $\sigma$ away from our result, so we conclude that the apsidal motion can only be much slower, with a period probably longer than a millenium. If one imposes $\omega_{1}=332.5^{\circ}$, the fit of the CORAVEL observations is clearly worse, with an rms scatter of the residuals of $3.83 \mathrm{~km} \mathrm{~s}^{-1}$ instead of $2.74 \mathrm{~km} \mathrm{~s}^{-1}$ (for both components); the difference is more visible on the $R V$ curve of the primary $\left(\sigma_{\text {res }}=3.89 \mathrm{~km} \mathrm{~s}^{-1}\right.$ instead of 2.35). Combining all published data with the CORAVEL ones, and after a correction $\Delta R V=-1.14 \mathrm{~km} \mathrm{~s}^{-1}$ to the latter for a better consistency, we obtain a very good curve with $\omega_{1}=314.46 \pm 1.44^{\circ}$ (Fig. A.2).

Fortunately, this system has a rather good Hipparcos parallax of $\pi=7.75 \pm 0.76$ mas, so that the radii of its components can be estimated. From the observed apparent magnitude $V_{1+2}=6.107$ (Rufener 1988) and from the magnitude difference $\Delta V=1.2$ (Abt et al. 1968) one gets the individual apparent magnitudes $V_{1}=6.42$ and $V_{2}=7.62$ which give the absolute magnitudes $M_{V 1}=0.87$ and $M_{V 2}=2.07$ using the Hipparcos parallax. From the spectral types A3 and A8 proposed by Abt et al. (1968), a first guess of the effective temperatures is given by the calibration of Hauck (1994): $T_{\text {eff } 1}=8275 \mathrm{~K}$ and $T_{\text {eff } 2}=7532 \mathrm{~K}$. Another guess can be done from the $(B 2-G)$ index of Geneva photometry, according to the calibration of Hauck \& North (1993): one has first to subtract the typical Geneva colours of the companion (assuming an $\mathrm{A} 8 \mathrm{~V}$ star) to the observed ones in order to get $(B 2-G)_{1}=-0.455$, which corresponds to $T_{\text {eff } 1}=8043 \mathrm{~K}$. Note that $(B 2-G)_{1}$ is not very sensitive to the assumption made on the companion, since it differs by only $0.023 \mathrm{mag}$ from the observed value $(B 2-G)_{1+2}=-0.432$. Adopting this effective temperature for the primary, an interpolation in the evolutionary tracks of Schaller et al. (1992) for an overall solar metallicity yields the physical parameters listed in Table A.7. It is interesting to notice that the mass ratio obtained in this way is $q=0.776 \pm 0.049$, which is compatible to better than one sigma with the dynamical mass ratio $q_{\mathrm{dyn}}=0.737 \pm 0.008$.

Also listed in Table A.7 are the radii estimated from the CORAVEL projected rotational velocities assuming a negligible Zeeman broadening, from the spin period (synchronization makes it equal to the orbital one) and from $i=66^{\circ}$. The latter value is obtained from $M_{1} \sin ^{3} i=1.733 \pm 0.030$ with the mass of the primary interpolated in the evolutionary tracks. It is almost identical with $i=67^{\circ}$ proposed by Abt et al. (1968). The radii obtained through the projected rotational velocities are compatible with those obtained from the Hipparcos luminosity and photometric effective temperatures, in the sense that error bars overlap. The agreement is perfect for the secondary, but much less satisfactory for the primary, even though the difference is less than twice the largest sigma. An attempt has been made to impose the dynamical mass ratio $q_{\text {dyn }}=0.737$ and interpolate in the evolutionary tracks the pair of stars whose magnitude difference is compatible with it. Maintaining the assumption of an $\mathrm{A} 8 \mathrm{~V}$ companion, we get in this way $\Delta V=1.54$ and $M_{1}=2.30 \pm .09, M_{2}=1.69 \pm .08 M_{\odot}, R_{1}=3.42 \pm 0.45$, $R_{2}=1.86 \pm 0.25 R_{\odot}$. The magnitude difference appears a bit large compared with the estimate of Abt et al. (1968) and the radius of the primary turns out to be even larger, making the discrepancy more severe compared to the radius estimated from the rotational velocity.

The number of CORAVEL measurements is too small to conclude about the possible variability of the depth and width of the correlation dip of the primary.

\section{A.11. $H D 105680\left(=B D+23^{\circ} 2423=\right.$ Renson 30570)}

This star was listed A3p SrSi? by Bertaud (1959), which motivated its inclusion in the sample, and as A3-F2 by Bertaud \& Floquet (1967). The radial-velocity curve is very well defined (see Fig. A.2). We secured 42 points over an interval of 2966 days. In spite of a relatively large $v \sin i$, the rms scatter of 
the residuals is small. Unfortunately, the classification remains ambiguous; $\Delta(V 1-G)=0.004$ suggests a mild peculiarity, but it is not large enough to exclude that it may be an Am star instead of an Ap.

\section{A.12. $H D 138426\left(=B D-18^{\circ} 4088=\right.$ Renson 39420$)$}

This poorly known star has been classified $\mathrm{Ap} \operatorname{SrCr}(\mathrm{Eu})$ by Houk \& Smith-Moore (1988). Its photometric peculiarity is just significant in the Geneva system $(\Delta(V 1-G)=0.010)$ and it is clearly an SB1 binary with a relatively short period. The $v \sin i$ is very small $\left(<2.4 \mathrm{~km} \mathrm{~s}^{-1}\right)$ and neither the depth nor the width of the correlation dip seems to vary. Figure A.2 shows a phase diagram of the radial velocities. The residual scatter is rather large, but the most discrepant points (at phases 0.48 and 0.56) were observed in the run of March 1997 where technical problems prevented the data to be registered on tape, so that it has not been possible to evaluate their quality.

\section{A.13. $H D 184471\left(=B D+32^{\circ} 3471=\right.$ Renson 50890)}

This star was classified A9 SrCrEu by Bertaud \& Floquet (1974). A total of 36 measurements have been made over almost 3500 days (Table 1 ), which clearly define a 429-day period (see Fig. A.2). The residuals are very small thanks to a small $v \sin i\left(<2 \mathrm{~km} \mathrm{~s}^{-1}\right)$ and a well contrasted dip.

\section{A.14. $H D 188854\left(=B D+46^{\circ} 2807=\right.$ Renson 52220)}

Ap or Am, according to different authors, its spectral type is not well determined. HD 188854 was listed as A7 CrEu by Bertaud \& Floquet (1974), but also as A5-F0 (Bertaud \& Floquet 1967). No $\Delta a$ photometry has been published for it, and the Geneva index $\Delta(V 1-G)=-0.002$ does not allow us to conclude, especially as it is among the coolest existing Ap stars. The radial-velocity curve is well determined with a $\sigma(\mathrm{O}-\mathrm{C})$ of $0.51 \mathrm{~km} \mathrm{~s}^{-1}$ only (see Fig. A.2).

\section{A.15. HD $200405(=B D+4703256=$ Renson 55830 $)$}

This A2 $\mathrm{SrCr}$ (Osawa 1965) star had already been announced as having the shortest orbital period known among all Bp and Ap stars (North 1994), with a period of only 1.635 days. A survey of the literature has not denied this claim: the few binaries with a period shorter than 3 days in Renson's (1991) catalogue either owe their spectral peculiarity to another physical cause like in situ nucleosynthesis (HD 93030, an "OBN" star according to Schönberner et al. 1988 and HD 49798, an O6 He star), or are misclassified (HD 25833, a normal B4V star according to Gimenez \& Clausen 1994), or do not have a typical Bp, Ap peculiarity (HD 124425, F7 MgCaSr in Renson's catalogue; HD 159876, F0IIIp in the Hipparcos Input Catalogue but A5F1 $\delta$ Del? in Renson's, and Am, A7/A9/F3 according to Abt \& Morrell 1995); finally, the A2 CrEu star HD 215661B is not a binary: only the A component of this visual system is an Algoltype binary.
HD 200405 is a bona fide Ap star also from the photometric point of view: Geneva photometry shows it is peculiar, with $\Delta(V 1-G)=0.021$, and Maitzen's peculiarity index $\Delta a=0.038$ on average (Schnell \& Maitzen 1995).

The inclination angle $i$ of the orbital plane of HD 200405 must be very small, according to the value of $a_{1} \sin i$ and of the mass function (Table A.1), unless the companion is a brown dwarf. The radial-velocity curve is shown in Fig. A.2. The orbit is circular $(e=0)$. This object is especially interesting, since it is exceptional: all other binaries with a magnetic Ap component have orbital periods longer than 3 days. If tidal effects tend to wash out the chemical peculiarity of the components, as suggested by this lower limit, then one has to explain how HD 200405 has been able to remain an Ap star in spite of significantly large tides.

Another way to interpret this radial-velocity curve would be to assume that HD 200405 has a very small, highly contrasted spot with enhanced abundance of iron-peak elements (whose lines are selected by the CORAVEL mask); in such a case, rotation alone might be responsible for a sinusoidal curve, if both the inclination $i$ of the rotational axis and the angle between the rotation and spot axes are such that the spot remains visible during the whole cycle. However, such a situation appears extremely improbable, since one does not see any variation in the intensity of the correlation dip, nor in its width or depth, which should occur because of the varying aspect of the spot. Likewise, the radial velocity of the $\mathrm{H}_{\alpha}$ line measured once with ELODIE is compatible with the CORAVEL $R V$ curve, while one would rather expect it to remain at the "systemic" velocity. Furthermore, the spot hypothesis would imply an apparent $v \sin i \approx 0 \mathrm{~km} \mathrm{~s}^{-1}$ (the radial velocity of every point in the spot being practically the same), while we obtain $v \sin i=9.5 \pm 0.4$ and $7.9 \pm 0.2 \mathrm{~km} \mathrm{~s}^{-1}$ using respectively CORAVEL and ELODIE: a small spot could never give rise to such a high value (the effect of the magnetic field has been removed in the ELODIE estimate). Therefore, HD 200405 holds the record of the shortest orbital period known.

\section{A.16. $H D 216533\left(=B D+58^{\circ} 2497=\right.$ Renson 59810)}

This A1 SrCr star (Osawa 1965) was already known as an SB1 system. Floquet (1979) found an orbital period of 16.03 days, using the radial velocity of the $\mathrm{Ca}$ II $\mathrm{K}$ line.

A total of 48 measurements have been made over almost 6225 days. The 16.03-day period does not fit at all our radial velocities. We find a much longer period $P=1413$ days (see Fig. A.2), which should be considered as more reliable. It seems that Floquet was too confident in her assumption of an homogeneous distribution of ionized calcium on the surface of the star, and that the $R V$ variation she observed was in fact due to a spot. The rotational period of this star, 17.2 days, is indeed very close to the "orbital" one found by Floquet (1979), although not identical.

Acknowledgements. This work was supported in part by the Swiss National Fondation for Scientific Research. The reduction of the data were made by the late Dr. Antoine Duquennoy and by SU. We thank the numerous observers who have contributed to this survey, 
especially Dr. J.-C. Mermilliod and Mr. Bernard Pernier. We also thank Dr. Noël Cramer, who had initiated the CORAVEL measurements of some bright Ap stars. We thank Dr. Thierry Forveille for having shared his ORBIT code for orbital elements determination from visual and RV data. This research has made use of the SIMBAD database, operated at CDS, Strasbourg, France. It was supported by the Swiss National Science Foundation.

\section{References}

Abt, H. A. 1953, PASP, 65, 274

Abt, H. A., Conti, P. S., Deutsch, A. J., \& Wallerstein, G. 1968, ApJ, 153,177

Abt, H. A., \& Levy, S. G. 1985, ApJS, 59, 229

Abt, H. A., \& Morrell, N. I. 1995, ApJS, 99, 135

Abt, H. A., \& Snowden, M. S. 1973, ApJS, 25, 137

Adelman, S. J., Pi, C.-L. M., \& Rayle, K. 1998, A\&AS, 133, 197

Aikman, G. C. L. 1976, Publ. Dominion Astrophys. Obs., 14, 379

Babcock, H. W. 1947, ApJ, 105, 105

Babcock, H. W. 1958, ApJS, 3,141

Babel, J., \& North, P. 1997, A\&A, 325, 195

Babel, J., North, P., \& Queloz, D. 1995, A\&A, 303, L5

Balega, I. I., \& Balega, Yu. Yu. 1987, SvAL, 13, 208

Balega, I. I. Balega, Yu. Yu., \& Vasyuk, V. A. 1989, Astrofiz. Issled. Izv. Spets. Astrofiz. Obs., 28, 107

Baranne, A., Mayor, M., \& Poncet, J.-L. 1979, Vistas Astron., 23, 279

Baranne, A., Queloz, D., Mayor, M., et al. 1996, A\&AS, 119, 373

Barzova, I. S., \& Iliev, I. K. 1988, in Magnetic stars, ed. Yu. V. Glagolevskij, \& I. M. Kopylov (Nizhnij Arkhyz), 107

Batten, A. H., Flechter, J. M., \& MacCarthy, D. G. 1989, PDAO, 17, 1

Benz, W., \& Mayor, M. 1981, A\&A, 93, 235

Benz, W., \& Mayor, M. 1984, A\&A, 138, 183

Bertaud, C. 1959, J. Obs., 42, 45

Bertaud, C. 1965, J. Obs., 48, 211

Bertaud, C., \& Floquet, M. 1967, J. Obs., 50, 425

Bertaud, C., \& Floquet, M. 1974, A\&AS, 16, 71

Bidelman, W. P. 1981, AJ, 86, 553

Bidelman, W. P., \& McConnell, D. J. 1973, AJ, 78, 687

Bonsack, W. K. 1976, ApJ, 209, 160

Borra, E. F., \& Landstreet, J. D. 1977, ApJ, 212, 141

Breiman, L. 1973, Statistics with a view towards applications (Houghton Mifflin Company, Boston)

Budaj, J. 1995, in: Stellar surface structure - Poster proceedings, ed. K. G. Strassmeier (University of Vienna), IAU Symp., 176, 147

Budaj, J. 1996, A\&A, 313, 523

Budaj, J. 1997, A\&A, 326, 655

Burki, G. 2002, Geneva photometric database http://obswww. unige.ch/gcpd/ph13.html

Cameron, R. C. 1966, Georgetown Obs. Monogr., 21

Catalano, F. A., \& Leone, F. 1994, A\&AS, 108, 595

Catalano, F. A., Leone, F., \& Kroll, R. 1998, A\&AS, 129, 463

Catalano, F. A., Renson, P., \& Leone, F. 1993, A\&AS, 98, 269

Catalano, F. A., \& Renson, P. 1997, A\&AS, 121, 57

Cowley, A., Cowley, C., Jaschek, M., \& Jaschek, C. 1969, AJ, 74, 375

Debernardi, Y. 2002, thesis No. 3362, Geneva University (this thesis is available upon request from the author, yves.debernardi@obs.unige.ch)

Debernardi, Y., Mermilliod, J.-C., Carquillat, J.-M., \& Ginestet, N. 2000, A\&A, 354, 891

Duquennoy, A., \& Mayor, M. 1991, A\&A, 248, 485

Edvardsson, B., Andersen, J., Gustafsson, B., et al. 1993, A\&A, 275, 101

ESA 1997, The HIPPARcos and Tycho Catalogues, ESA SP-1200
Fehrenbach, C., Duflot, M., Mannone, C., Burnage, R., \& Genty, V. 1997, A\&AS, 124, 255

Floquet, M. 1979, A\&A, 77, 263

Gerbaldi, M., Floquet, M., \& Hauck, B. 1985, A\&A, 146, 341

Gimenez, A., \& Clausen, J. V. 1994, A\&A, 291, 795

Ginestet, N., Carquillat, J. M., Jaschek, C., \& Jaschek, M. 1997, A\&AS, 123, 135

Ginestet, N., Griffin, R. F., Carquillat, J. M., \& Udry, S. 1999, A\&A, 140,279

Gray, R. O., \& Garrisson, R. F. 1989, ApJS, 70, 623

Grenier, S., Baylac, M.-O., Rolland, L., et al. 1999, A\&A, 137, 451

Harper, W. E. 1931, Publ. Dominion Astrophys. Obs., 4, 179

Hartkopf, W. I., Mason, B. D., \& McAlister, H. A. 1996, AJ, 111, 370

Hartkopf, W. I., McAlister, H. A., \& Franz, O. G. 1992, AJ, 104, 810

Hartkopf, W. I., McAlister, H. A., Mason, B. D., et al. 1994, AJ, 108, 2299

Hauck, B. 1985, in Calibration of fundamental stellar quantities, ed. D. S. Hayes, L. E. Pasinetti, \& A. G. D. Philip, IAU Symp., 111, 271

Hauck, B. 1994, in The MK process at 50 years: A powerful tool for astrophysical insight, ed. C. J. Corbally, R. O. Gray, \& R. F. Garrison, ASP Conf. Ser., 60, 157

Hauck, B., \& North, P. 1993, A\&A, 269, 403

Houk, N., \& Smith-Moore, M. 1988, Michigan catalogue of twodimensional spectral types for the HD stars, vol. 4, University of Michigan, Ann Arbor

Huchra, J. 1972, ApJ, 174, 435

Iliev, I. K., Barzova, I. S., \& Peeva, P. S. 1992, in Stellar magnetism, ed. Yu. V. Glagolevskij, \& I. I. Romanyuk (NAUKA, Saint-Petersburg), 145

Jaschek, C., \& Gomez, A. E. 1970, PASP, 82, 809

Jaschek, C., Jaschek, M., Gomez, A. E., \& Grenier, J. S. 1983, A\&A, 127,1

Kurtz, D. W. 1982, MNRAS, 200, 807

Kurtz, D. W. 1991, MNRAS, 249, 468

Leone, F., \& Catanzaro, G. 1999, A\&A, 343, 273

Leroy, J. L. 1995, A\&AS, 114, 79

Levato, H., Malaroda, S., Morrell, N., \& Solivella, G. 1987, ApJS, 64, 487

Lloyd, C., Stickland, D., \& Walborn, N. R. 1995, PASP, 107, 1030

Lucke, P. B. 1978, A\&A, 64, 367

Lucy, L. B., \& Sweeney, M. A. 1971, AJ, 76, 544

Lutz, T. E., \& Kelker, D. H. 1973, PASP, 85, 573

Maitzen, H. M. 1976, A\&A, 51, 223

Maitzen, H. M., \& Pavlovski, K. 1987, A\&AS, 71, 441

Maitzen, H. M., Pressberger, R., \& Paunzen, E. 1998, A\&AS, 128, 573

Maitzen, H. M., \& Seggewiss, W. 1980, A\&A, 83, 328

Maitzen, H. M., \& Vogt, N. 1983, A\&A, 123, 48

Martin, C., \& Mignard, F. 1998, A\&A, 330, 585

Mathys, G. 1988, in Elemental abundance analyses, ed. S. J. Adelman, \& T. Lanz, Institut d'Astronomie de l'Université de Lausanne, 101

Mathys, G. 1994, A\&AS, 108, 547

Mathys, G., \& Hubrig, S. 1995, A\&A, 293, 810

Mathys, G., \& Hubrig, S. 1997, A\&AS, 124, 475

Mathys, G., Hubrig, S., Landstreet, J. D., Lanz, T., \& Manfroid, J. 1997, A\&AS, 123, 353

Mathys, G., \& Lanz, T. 1990, A\&A, 230, L21

Mayor, M. 1980, A\&A, 87, L1

McAlister, H. A., Hendry, E. M., Hartkopf, W. I., Campbell, B. G., \& Fekel, F. C. 1983, ApJS, 51, 309

McAlister, H. A., Hartkopf, W. I., Hutter, D. J., \& Franz, O. G. 1987, AJ, 93, 688 
McAlister, H. A., Hartkopf, W. I., \& Franz, O. G. 1990, AJ, 99, 965

Mermilliod, J.-C., \& Mayor, M. 1996, in Cool stars, stellar systems and the sun, Ninth Cambridge workshop, ed. R. Pallavicini, \& A. K. Dupree, ASP Conf. Ser., 109, 373

Mermilliod, J.-C., Mermilliod, M., \& Hauck, B. 1997, A\&AS, 124, 349

Mkrtichian, D. E., Samus, N. N., \& Gorynya, N. A. 1997, in Stellar magnetic fields, ed. Yu. V. Glagolevskij, \& I. I. Romanyuk (Moscow, Russian Academy of Sciences), 185

Mkrtichian, D. E., Samus, N. N., Gorynya, N. A., et al. 1998, IBVS, 4564

Mkrtichian, D. E., Samus, N. N., Gorynya, N. A., North, P., \& Hatzes, A. P. 1999, in Precise radial velocities, ed. J. B. Hearnshaw, \& C. D. Scarfe, ASP Conf. Ser., 185, 331

Moon, T., \& Dworetsky, M. 1985, MNRAS, 217, 305

Musielok, B. 1976, Astron. Nachrichten, 297, 169

North, P. 1993, in Peculiar versus normal phenomena in A-type and related stars, ed. M. M. Dworetsky, F. Castelli, \& R. Faraggiana, ASP Conf. Ser., 44, 577

North, P. 1994, in The 25th workshop and meeting of European Working Group on CP stars, ed. I. Jankovicz, \& I. Vincze (Szombathely), 3

North, P. 1998, A\&A, 334, 181

North, P. 1998, Highlights Astron., 11B, 657

North, P., Babel, J., \& Lanz, T. 1988, IBVS, 3155

North, P., \& Duquennoy, A. 1991, A\&A, 244, 335

North, P., Carquillat, J.-M., Ginestet, N., Carrier, F., \& Udry, S. 1998, A\&AS, 130, 223 (Paper I)

North, P., Jaschek, C., Hauck, B., et al. 1997, ESA SP-402, 239

Northcott, R. J. 1948, AJ, 53, 116

Olsen, E. H. 1983, A\&AS, 54, 55

Olsen, E. H. 1994, A\&AS, 106, 257

Osawa, K. 1965, Ann. Tokyo Astron. Obs., 2nd series IX, 123

Pilachowski, C. A., Bonsack, W. K., \& Wolff, S. C. 1974, A\&A, 37, 275

Pont, F. 1997, Thesis No. 2922, Geneva University, 130 (this part of the thesis available upon request from the authors)
Preston, G. W. 1967, ApJ, 150, 871

Preston, G. W. 1970, ApJ, 160, 1059

Preston, G. W. 1971, ApJ, 164, 309

Preston, G. W., \& Wolff, S. C. 1970, ApJ, 160, 1071

Rakosch, K. D., \& Fiedler, W. 1978, A\&AS, 31, 83

Renson, P. 1978, A\&A, 63, 125

Renson, P. 1991, Catalogue Général des Étoiles Ap et Am, Institut d'Astrophysique - Université de Liège

Rice, J. B. 1988, A\&A, 199, 299

Rufener, F. 1988, Catalogue of Stars measured in the Geneva Observatory Photometric system (fourth edition), Observatoire de Genève

Ryabchikova, T. 1998, Contrib. Astron. Obs. Skalnaté Pleso, 27, 319

Schaller, G., Schaerer, D., Maeder, A., \& Meynet, G. 1992, A\&AS, 96, 269

Schnell, A., \& Maitzen, H. M. 1995, IBVS, 4175

Scholz, G. 1978, Astron. Nachr., 299, 81

Scholz, G., Hildebrandt, G., Lehmann, H., \& Glagolevskij, Yu. V. 1997, A\&A, 325, 529

Scholz, G., \& Lehmann, H. 1988, Astron. Nachr., 309, 33

Schönberner, D., Herrero, A., Becker, S., et al. 1988, A\&A, 197, 209

Stibbs, D. W. N. 1950, MNRAS, 110, 395

Stickland, D. J., \& Lloyd, C. 1994, Obs., 114, 41

Udry, S., Mayor, M., \& Queloz, D. 1999, ASP Conf. Ser., 185, IAU Coll., 170, 367

van den Heuvel, E. P. J. 1971, A\&A, 11, 461

Vetö, B., Schöneich, W., \& Rustamov, Yu. S. 1980, Astron. Nachrichten, 301, 317

Wade, G. A., North, P., Mathys, G., \& Hubrig, S. 1996, A\&A, 314, 491

Wade, G. A., Debernardi, Y., Mathys, G., et al. 2000, A\&A, 361, 991

Wade, G. A., Mathys, G., \& North, P. 1999, A\&A, 347, 164

Wolff, S. C. 1974, PASP, 86,179

Wolff, S. C. 1975, ApJ, 202, 127

Zahn, J.-P. 1977, A\&A, 57, 383

Zahn, J.-P. 1989, A\&A, 220, 112

Zahn, J.-P., \& Bouchet, L. 1989, A\&A, 223, 112 